\title{
ON THE DISTANCE BETWEEN THE EMPIRICAL PROCESS AND ITS CONCAVE MAJORANT IN A MONOTONE REGRESSION FRAMEWORK
}

\section{SUR LA DISTANCE ENTRE LE PROCESSUS EMPIRIQUE ET SON MAJORANT CONCAVE DANS UN MODÈLE DE RÉGRESSION MONOTONE}

\author{
Cécile DUROT $^{\mathrm{a}, *}$, Anne-Sophie TOCQUET ${ }^{\mathrm{b}}$ \\ ${ }^{a}$ Laboratoire de statistiques, bâtiment 425, Université Paris Sud, 91405 Orsay cedex, France \\ ${ }^{\mathrm{b}}$ UPRESA CNRS 8071, génopole d'Évry, Université d'Évry Val d'Essonne, \\ 523, place des terrasses de l'Agora, 91000 Évry, France
}

Received 19 September 2000, revised 24 July 2001

ABSTRACT. - We consider a regression model where the unknown regression function $f$ is known to be decreasing and defined over $[0,1]$. Our aim is to study the distance between the empirical process $F_{n}$ and its least concave majorant $\widehat{F}_{n}$. We obtain the asymptotic distributions of both $\left.\widehat{F}_{n}(x)-F_{n}(x), x \in\right] 0,1$, and the $\mathbb{L}_{q}$-distance between $\widehat{F}_{n}$ and $F_{n}$. These distributions depend on the distribution of the process $\widehat{X}-X$ where $X$ is a standard two-sided Brownian motion with parabolic drift and $\widehat{X}$ is the least concave majorant of $X$. Some properties of this process are described. Finally we provide the order of magnitude of the supremum distance between $\widehat{F}_{n}$ and $F_{n}$.

() 2003 Éditions scientifiques et médicales Elsevier SAS

MSC: 62E20; 62G20

Keywords: Nonparametric regression; Isotonic regression; Brownian motion with parabolic drift; Least concave majorant; Central limit theorem

RÉSUMÉ. - Considérons un modèle de régression dans lequel la fonction de régression inconnue est définie sur $[0,1]$ et supposée décroissante. Nous étudions la distance entre le processus empirique $F_{n}$ associé à ce modèle et le plus petit majorant concave $\widehat{F}_{n}$ de $F_{n}$. Nous obtenons les lois asymptotiques des écarts, ponctuel et en distance $\mathbb{L}_{q}$, entre $\widehat{F}_{n}$ et $F_{n}$. Ces lois s'expriment en fonction de la loi du processus $\widehat{X}-X$ où $X$ est un mouvement Brownien standard

\footnotetext{
* Corresponding author.

E-mail addresses: cecile.durot@math.u-psud.fr (C. Durot), tocquet@ genopole.cnrs.fr (A.-S. Tocquet).
} 
sur $\mathbb{R}$ avec dérive parabolique et $\widehat{X}$ est le plus petit majorant concave de $X$. Nous énonçons quelques propriétés de ce processus. Nous donnons enfin l'ordre de grandeur de la distance uniforme entre $\widehat{F}_{n}$ et $F_{n}$.

( 2003 Éditions scientifiques et médicales Elsevier SAS

\section{Introduction}

We observe $y_{1}, \ldots, y_{n}$ according to the following model

$$
y_{i}=f\left(x_{i}\right)+\varepsilon_{i}, \quad i=1, \ldots, n
$$

where $x_{i}=i / n$, the $\varepsilon_{i}$ 's are independent and identically distributed random variables with mean zero and variance $\sigma^{2}$ and the regression function $f$ is assumed to be monotone - say decreasing - on $[0,1]$. Our aim is to study the asymptotic behaviour of $\widehat{F}_{n}-F_{n}$, where $F_{n}$ is the empirical process given by

$$
\forall t \in[0,1], \quad F_{n}(t)=\frac{1}{n} \sum_{i=1}^{n} y_{i} \mathbb{1}_{x_{i} \leqslant t}
$$

and $\widehat{F}_{n}$ is the least concave majorant of $F_{n}$. We first study the pointwise asymptotic distribution of $\widehat{F}_{n}-F_{n}$. It depends on the distribution of $\widehat{X}(0)$, defined as the least concave majorant at time zero of the standard two-sided Brownian motion with parabolic drift. We state in Theorem 4.1 that for every $x \in] 0,1\left[, \widehat{F}_{n}(x)-F_{n}(x)\right.$ converges at the $n^{2 / 3}$-rate to $C_{f} \widehat{X}(0)$, where $C_{f}$ is a normalizing constant that depends on both $f$ and $\sigma^{2}$. We are then interested in the global behaviour of $\widehat{F}_{n}-F_{n}$. In Theorem 5.1, it is stated that the order of magnitude of the supremum distance between $\widehat{F}_{n}$ and $F_{n}$ is $n^{-2 / 3}(\log n)^{2 / 3}$. Finally, we give in Theorem 5.2 the asymptotic distribution of the $\mathbb{L}_{q}$-distance between $\widehat{F}_{n}$ and $F_{n}$. It is shown that a centered version of $\int_{0}^{1}\left|\widehat{F}_{n}(t)-F_{n}(t)\right|^{q} d t$ converges at the $n^{(1+4 q) / 6}$-rate to a Gaussian variable.

The problem of studying the empirical process under order restrictions has been considered in the context of density estimation by Kiefer and Wolfowitz [5], Wang [10] and is, at the time of the writing of this paper, also studied by Kulikov and Lopuhaä [6]. In this framework, the authors have considered the empirical distribution function associated to a random sample whose underlying density is assumed to be decreasing. Wang has described the asymptotic distribution of the difference between the empirical distribution function and its least concave majorant at a given point. Kiefer, Wolfowitz, Kulikov and Lopuhaä have studied the distance between the empirical distribution function and its concave majorant in the global sense. Kiefer and Wolfowitz have proved that the least concave majorant of the empirical distribution function is asymptotically minimax estimator of the underlying concave distribution function. They have also proved that the supremum-distance between the empirical distribution function and its concave majorant is $\mathcal{O}_{\mathbb{P}}\left(n^{-2 / 3}(\log n)^{2 / 3}\right)$ (one can easily check that the proof of their Theorem 1 provides this rate). Kulikov and Lopuhaä have proved a central limit theorem for the $\mathbb{L}_{1}$-distance between the empirical distribution function and its concave majorant, which is close in spirit to our Theorem 5.2. Our Theorems 4.1 and 5.1 can be compared 
to the results of Kiefer, Wolfowitz and Wang. The pointwise asymptotic distribution we obtain in regression framework is similar to the one obtained by Wang, appart from a normalizing constant. Moreover, the upper bounds $n^{-2 / 3}(\log n)^{2 / 3}$ for the supremum distances are the same in both frameworks. In Theorem 5.1, we also provide a lower bound that proves that the supremum distance is exactly of order $n^{-2 / 3}(\log n)^{2 / 3}$.

It is worth noticing that all the asymptotic distributions mentioned above depend on a process $\widehat{X}-X$ defined as follows: for every $\eta \in \mathbb{R}, X(\eta)=-\eta^{2}+W(\eta)$ where $W$ is a standard two-sided Brownian motion, and $\widehat{X}$ is the least concave majorant of $X$. Functionals of Brownian motion with parabolic drift usually appear in asymptotic distribution of estimators obtained under order restrictions. For instance, Prakasa Rao [7], Brunk [1], Huang and Wellner [4] have studied the pointwise asymptotic distribution of the isotonic estimator of a monotone density and of a monotone regression function. They have proved that, when properly normalized, these estimators converge in distribution to the location of the maximum of $X$ (that is distributed as half the slope of $\widehat{X}$ at time zero). Groeneboom et al. [3] and Durot [2] have proved, in density and regression framework respectively, a central limit theorem for the $\mathbb{L}_{1}$-distance between the isotonic estimator and the function to be estimated. In both cases, asymptotic expectation and variance depend on a location process related to Brownian motion with parabolic drift.

The paper is organized as follows. Some properties of least concave majorants are given in Section 2. In Section 3 we describe some properties of the canonical process $\widehat{X}-X$ the asymptotic distribution of $\widehat{F}_{n}-F_{n}$ depends on. Pointwise asymptotic distribution of $\widehat{F}_{n}-F_{n}$ is given in Section 4. Finally, global behaviour of $\widehat{F}_{n}-F_{n}$ is studied in Section 5.

\section{Some properties of least concave majorants}

In this section, $I$ denotes an interval in $\mathbb{R}, Z$ denotes a process indexed by $I$ and $\widehat{Z}$ denotes the least concave majorant of $\{Z(\eta), \eta \in I\}$. The first lemma of this section summarizes properties of least concave majorants that have been used by Prakasa Rao [7] and others.

LEMMA 2.1. - For every $t \in I, a>0, b \in \mathbb{R}$, the least concave majorant at time $t$ of $\{a Z(\eta)-b, \eta \in I\}$ is $a \widehat{Z}(t)-b$ and the least concave majorant at time $t$ of $\{Z(\eta)-b \eta, \eta \in I\}$ is $\widehat{Z}(t)-b t$. Moreover, we have the following property of change of variable: if $J$ denotes $\{\eta, a \eta+b \in I\}$ then for every $t \in J, a \neq 0$ and $b \in \mathbb{R}$, the least concave majorant at time $t$ of $\{Z(a \eta+b), \eta \in J\}$ is $\widehat{Z}(a t+b)$.

Proof. - We only prove here the first property. The last two properties can be proved in the same way. Let $\widehat{G}$ be the least concave majorant of $\{G(\eta), \eta \in I\}$, where $G(\eta)=$ $a Z(\eta)-b$. Then $\eta \mapsto(\widehat{G}(\eta)+b) / a$ is concave and above $Z$ so $(\widehat{G}(t)+b) / a \geqslant \widehat{Z}(t)$ for all $t \in I$. Likewise, $\eta \mapsto a \widehat{Z}(\eta)-b$ is concave and above $G$ so $a \widehat{Z}(t)-b \geqslant \widehat{G}(t)$ for all $t \in I$, which proves that $a \widehat{Z}(t)-b=\widehat{G}(t)$ for every $t$.

The following result will be repeatedly used in the paper. An interesting consequence of this result is that the supremum distance between least concave majorants of two processes converges to zero whenever the supremum distance between the processes themselves converges to zero. 
LEMMA 2.2. - Let $Z$ and $Z^{\prime}$ be processes indexed by $I$ and let $\widehat{Z}$ and $\widehat{Z}^{\prime}$ denote the least concave majorants of $Z$ and $Z^{\prime}$ respectively. Then,

$$
\sup _{\eta \in I}\left|\widehat{Z}(\eta)-\widehat{Z}^{\prime}(\eta)\right| \leqslant \sup _{\eta \in I}\left|Z(\eta)-Z^{\prime}(\eta)\right| \text {. }
$$

Proof. - Fix $x \in I$ and assume without loss of generality that $\widehat{Z}(x) \geqslant \widehat{Z}^{\prime}(x)$. Assume first $\widehat{Z}(x)=Z(x)$. Then $\left|\widehat{Z}(x)-\widehat{Z}^{\prime}(x)\right| \leqslant Z(x)-Z^{\prime}(x)$, which is less than or equal to the supremum distance between $Z$ and $Z^{\prime}$. Assume now $\widehat{Z}(x)>Z(x)$. Then there exist $u<x$ and $v>x$ such that $\widehat{Z}(u)=Z(u), \widehat{Z}(v)=Z(v)$ and $\widehat{Z}$ is affine on $[u, v]$ (that is $\widehat{Z}(t)=\widehat{Z}(u)+(t-u) \frac{\widehat{Z}(v)-\widehat{Z}(u)}{v-u}$ for every $\left.t \in[u, v]\right)$. Also, $t \mapsto \widehat{Z}(t)-\widehat{Z}^{\prime}(t)$ is convex on $[u, v]$ and therefore,

$$
\widehat{Z}(x)-\widehat{Z}^{\prime}(x) \leqslant \max \left\{\widehat{Z}(u)-\widehat{Z}^{\prime}(u), \widehat{Z}(v)-\widehat{Z}^{\prime}(v)\right\} \leqslant \sup _{\eta \in I}\left|Z(\eta)-Z^{\prime}(\eta)\right| .
$$

We thus have

$$
\left|\widehat{Z}(x)-\widehat{Z}^{\prime}(x)\right| \leqslant \sup _{\eta \in I}\left|Z(\eta)-Z^{\prime}(\eta)\right|
$$

for every $x \in I$, which proves the lemma.

\section{The canonical process}

Both pointwise and global asymptotic distributions of the distance between the empirical process $F_{n}$ defined by (1.2) and its least concave majorant $\widehat{F}_{n}$ depend on a canonical process $\widehat{X}-X$ defined as follows: for every $\eta \in \mathbb{R}, X(\eta)=-\eta^{2}+W(\eta)$ where $W$ is a standard two-sided Brownian motion, and $\widehat{X}$ is the least concave majorant of $X$. The aim of this section is to describe properties of this canonical process. We first state a stationarity property.

LEMMA 3.1. - For every $t \in \mathbb{R}, \widehat{X}(t)-X(t)$ is identical in distribution to $\widehat{X}(0)$.

Proof. - By Lemma 2.1, $\widehat{X}(t)-X(t)$ is the least concave majorant at time zero of the process $\left\{-(\eta+t)^{2}+W(\eta+t)+t^{2}-W(t), \eta \in \mathbb{R}\right\}$. It is thus identical in distribution to the least concave majorant at time zero of the process $\left\{-(\eta+t)^{2}+W(\eta)+t^{2}, \eta \in \mathbb{R}\right\}$, that is equal to $\widehat{X}(0)$ (see the second property in Lemma 2.1).

We now prove in Lemma 3.2 that the least concave majorant $\widehat{X}$ at a given point $t$ is essentially unchanged if the process $X$ is restricted to some long enough bounded interval centered at $t$. The increments of Brownian motion are independent and the difference between $X(t)$ and the least concave majorant at time $t$ of $\{X(\eta), \eta \in$ $[t-2 c, t+2 c]\}$ only depends on the increments of the Brownian motion $W$ on $[t-2 c, t+2 c]$. It thus can be shown from Lemma 3.2 that $\widehat{X}-X$ is a mixing process.

LEMMA 3.2. - Let c be some positive real number and for every $t \in \mathbb{R}$, let $\widehat{X}_{c}^{(t)}$ denote the least concave majorant of $\{X(\eta), \eta \in[t-2 c, t+2 c]\}$. For all $t \in \mathbb{R}$,

$$
\mathbb{P}\left(\widehat{X}(t) \neq \widehat{X}_{c}^{(t)}(t)\right) \leqslant 4 \exp \left(-c^{3} / 2\right) .
$$


Proof. - Let $R(c)$ be defined by

$$
R(c)=\mathbb{P}\left(\exists u<a-c, \exists v>a+c, X(u)+\frac{X(u)-X(v)}{u-v}(a-u)>X(a)\right) .
$$

Then $R(c)$ does not depend on $a$ since $\{W(t+a)-W(a), t \in \mathbb{R}\}$ is identical in distribution to $\{W(t), t \in \mathbb{R}\}$ for every fixed $a \in \mathbb{R}$. We have

$$
\mathbb{P}\left(\widehat{X}(t) \neq \widehat{X}_{c}^{(t)}(t)\right) \leqslant \mathbb{P}(A \cup B),
$$

where $A$ is the event that there exist some $u<t, v>t+2 c$ such that the line segment joining $(u, X(u))$ and $(v, X(v))$ is above $(t+c, X(t+c))$ and $B$ is the event that there exist some $u<t-2 c, v>t$ such that the line segment joining $(u, X(u))$ and $(v, X(v))$ is above $(t-c, X(t-c))$. Setting $a=t+c$ yields $\mathbb{P}(A)=R(c)$. Likewise, setting $a=t-c$ yields $\mathbb{P}(B)=R(c)$. Therefore, $\mathbb{P}\left(\widehat{X}(t) \neq \widehat{X}_{c}^{(t)}(t)\right) \leqslant 2 R(c)$. (Note that the above arguments leading to the latter inequality are due to Prakasa Rao [7], see his Lemma 6.2.) Setting $a=0$ yields

$$
\mathbb{P}\left(\widehat{X}(t) \neq \widehat{X}_{c}^{(t)}(t)\right) \leqslant 2 \mathbb{P}(\{\exists u<-c, X(u)>0\} \quad \text { or }\{\exists v>c, X(v)>0\}) .
$$

Let $b$ and $c$ be positive numbers. Change of variable $v=c / u$ yields

$$
\mathbb{P}\left(\sup _{u>c}\left\{W(u)-b u^{2}\right\}>0\right) \leqslant \mathbb{P}\left(\sup _{v \in(0,1]}\left\{\frac{v^{2}}{\sqrt{c}} W\left(\frac{c}{v}\right)\right\}>b c^{3 / 2}\right) .
$$

But $\left\{v c^{-1 / 2} W(c / v), v \in \mathbb{R}\right\}$ is a standard two-sided Brownian motion (see e.g. Revuz and Yor [8], pp. 19-20) so

$$
\mathbb{P}\left(\sup _{u>c}\left\{W(u)-b u^{2}\right\}>0\right) \leqslant \mathbb{P}\left(\sup _{v \in[0,1]} W(v)>b c^{3 / 2}\right) .
$$

By exponential inequality (see e.g. Revuz and Yor [8], p. 52) we obtain

$$
\mathbb{P}\left(\sup _{u>c}\left\{W(u)-b u^{2}\right\}>0\right) \leqslant \exp \left(-b^{2} c^{3} / 2\right),
$$

and Lemma 3.2 follows.

We now state probability and covariance inequalities that imply existence and finiteness of $m_{q}$ and $k_{q}$, defined for $q>0$ by

$$
m_{q}=\mathbb{E}|\widehat{X}(0)|^{q} \quad \text { and } \quad k_{q}=\int_{0}^{\infty} \operatorname{cov}\left(|\widehat{X}(0)|^{q},|\widehat{X}(t)-X(t)|^{q}\right) d t .
$$

LEMMA 3.3. - For every $t>0$,

$$
\mathbb{P}(\widehat{X}(0)>t) \leqslant 4 \exp \left(-t^{3 / 2} / 2\right) .
$$


Moreover, for every $q>0$ there exists some positive constant $A_{q}$ such that for every $t \in \mathbb{R}$

$$
\left|\operatorname{cov}\left(|\widehat{X}(0)|^{q},|\widehat{X}(t)-X(t)|^{q}\right)\right| \leqslant A_{q} \exp \left(-\frac{|t|^{3}}{2^{10}}\right) .
$$

Therefore, for every $q>0$, the constants $m_{q}$ and $k_{q}$ as defined in (3.4) are finite.

Proof. - It follows from (3.3) that for all $t>0$,

$$
\mathbb{P}\left(\sup _{|\eta|>t^{1 / 2}}\{X(\eta)\}>0\right) \leqslant 2 \exp \left(-t^{3 / 2} / 2\right) .
$$

For all $\eta, X(\eta) \leqslant W(\eta)$ so it follows from scaling and symmetry of Brownian motion that

$$
\mathbb{P}\left(\sup _{|\eta| \leqslant t^{1 / 2}}\{X(\eta)\}>t\right) \leqslant 2 \mathbb{P}\left(\sup _{\eta \in[0,1]}\{W(\eta)\}>t^{3 / 4}\right),
$$

where by exponential inequality, the latter probability is less than or equal to $\exp \left(-t^{3 / 2} / 2\right)$. Inequality (3.5) now follows from the fact that the least concave majorant at time zero of the process $X$ is less than or equal to the supremum of $X$. Integrating (3.5) yields $m_{q}<\infty$ for all $q>0$.

Inequality (3.6) is an immediate consequence of finiteness of $m_{2 q}$ whenever $t=0$. We thus assume $t \neq 0$. For every $t \in \mathbb{R} \backslash\{0\}$ and $c>0$, let $\widehat{X}_{c}^{(t)}$ be defined as in Lemma 3.2. For the sake of simplicity we note $\widehat{X}_{c}^{(t)}(t)=\widehat{X}_{c}(t)$. Since for all $t, \widehat{X}_{c}(t)-X(t)$ is the least concave majorant at time zero of the process $\left\{-(\eta+t)^{2}+W(\eta+t)+t^{2}-\right.$ $W(t), \eta \in[-2 c, 2 c]\}$ (see Lemma 2.1), it only depends on the increments of $W$ between times $t-2 c$ and $t+2 c$. Increments of Brownian motion are independent so the random variables $\widehat{X}_{c}(t)-X(t)$ and $\widehat{X}_{c}(0)$ are independent whenever $c \leqslant|t| / 4$. In the sequel we set $c=|t| / 4$. Then,

$$
\operatorname{cov}\left(\left|\widehat{X}_{c}(0)\right|^{q},\left|\widehat{X}_{c}(t)-X(t)\right|^{q}\right)=0 .
$$

By Lemma 3.1, $\mathbb{E}|\widehat{X}(t)-X(t)|^{q}=m_{q}$ for all $t \in \mathbb{R}$. Since $X(t) \leqslant \widehat{X}_{c}(t) \leqslant \widehat{X}(t)$, by Cauchy-Schwarz inequality and Lemma 3.2 we have for all $l>0$

$$
\mathbb{E}\left|\widehat{X}_{c}(t)-\widehat{X}(t)\right|^{l} \leqslant m_{2 l}^{1 / 2} \mathbb{P}^{1 / 2}\left(\widehat{X}_{c}(t) \neq \widehat{X}(t)\right) \leqslant 2 m_{2 l}^{1 / 2} \exp \left(-c^{3} / 4\right) .
$$

We obtain (3.6) by using the following consequence of Cauchy-Schwarz inequality: if $X, Y, X^{\prime}$ and $Y^{\prime}$ are random variables in $\mathbb{L}_{2}$ then

$$
\left|\operatorname{cov}(X, Y)-\operatorname{cov}\left(X^{\prime}, Y^{\prime}\right)\right| \leqslant \mathbb{E}^{1 / 2}|X|^{2} \mathbb{E}^{1 / 2}\left|Y-Y^{\prime}\right|^{2}+\mathbb{E}^{1 / 2}\left|Y^{\prime}\right|^{2} \mathbb{E}^{1 / 2}\left|X-X^{\prime}\right|^{2} .
$$

Existence and finiteness of $k_{q}$ follows from (3.6).

\section{Pointwise asymptotic distribution of $\widehat{\boldsymbol{F}}_{\boldsymbol{n}}-\boldsymbol{F}_{\boldsymbol{n}}$}

In this section, we describe the asymptotic distribution of $\widehat{F}_{n}-F_{n}$ at a given point $x \in] 0,1\left[\right.$. We prove that, when properly normalized, $n^{2 / 3}\left(\widehat{F}_{n}(x)-F_{n}(x)\right)$ converges 
in distribution to $\widehat{X}(0)$ defined in Section 3. Apart from a normalizing constant that depends on $f$ and $\sigma^{2}=\mathbb{E}\left(\varepsilon_{1}^{2}\right)$, the asymptotic distribution we obtain is the same as the one obtained by Wang in the context of density estimation, see [10].

THEOREM 4.1. - Assume we are given the regression model (1.1), where $x_{i}=i / n$, the $\varepsilon_{i}$ 's are centered independent and identically distributed random variables and $f$ is a decreasing twice differentiable function with bounded second derivative. Let $F_{n}$ be the empirical process defined by (1.2) and let $\widehat{F}_{n}$ be the least concave majorant of $F_{n}$. If there exists some $p>3$ such that $\mathbb{E}\left|\varepsilon_{1}\right|^{p}$ is finite and if $\inf _{t \in[0,1]}\left|f^{\prime}(t)\right|>0$, then for every $x \in] 0,1[$,

$$
n^{2 / 3}\left(\widehat{F}_{n}(x)-F_{n}(x)\right) \stackrel{\mathcal{D}}{\longrightarrow} \sigma^{4 / 3}\left|\frac{f^{\prime}(x)}{2}\right|^{-1 / 3} \widehat{X}(0), \quad \text { as } n \rightarrow \infty,
$$

where $\sigma^{2}=\mathbb{E}\left(\varepsilon_{1}^{2}\right), \widehat{X}$ is the least concave majorant of the process $\left\{W(\eta)-\eta^{2}, \eta \in \mathbb{R}\right\}$ and $W$ is a standard two-sided Brownian motion.

The rest of this section is devoted to the proof of Theorem 4.1. Some of the ideas involved in this proof are close in spirit to Prakasa Rao's. Arguments used to obtain the asymptotic distribution of $n^{2 / 3}\left(\widehat{F}_{n}(x)-F_{n}(x)\right)$ are the following. We assume $\sigma=1$. First, we prove that $F_{n}$ can be approximated in distribution by some continuous process $G_{n}$ defined by

$$
\forall \eta \in[0,1], \quad G_{n}(\eta)=F(\eta)+W(\eta) / \sqrt{n},
$$

where $F(\eta)=\int_{0}^{\eta} f(s) d s$ and $W$ is a standard two-sided Brownian motion, see Lemma 4.1 below. The least concave majorant of $F_{n}$ can then be approximated in law by the least concave majorant $\widehat{G}_{n}$ of $G_{n}$. Next, we prove that the least concave majorant $\widehat{G}_{n}$ of $G_{n}$ at a given point $x$ is essentially unchanged if the process $G_{n}$ is restricted to some interval with length $4 c n^{-1 / 3}$ (where $c>0$ ) and centered at $x$, see Lemma 4.2 below. The result then follows from properties of least concave majorants described in Section 2.

For the sake of simplicity, we have assumed in Theorem 4.1 that $f$ satisfies global regularity conditions. However, it is worth noticing that the convergence result in Theorem 4.1 remains true under local regularity conditions. More specifically, the convergence result in Theorem 4.1 still holds under the following less restrictive conditions: $x_{i}=i / n, f$ is non-increasing on $[0,1]$ and continuously differentiable in a neighbourhood of $x, f^{\prime}(x)<0$, the $\varepsilon_{i}$ 's are i.i.d. with $\mathbb{E} \varepsilon_{i}=0$ and $\mathbb{E}\left|\varepsilon_{1}\right|^{p}<\infty$ for some $p>2$. To prove this, one can first prove in the same way as Lemma 4.2 that for large enough $c, \widehat{F}_{n}(x)$ is essentially identical to $\widehat{F}_{n, c}(x)$, the least concave majorant at time $x$ of the restricted process $\left\{F_{n}(\eta),|\eta-x| \leqslant 2 c n^{-1 / 3}\right\}$. More precisely, one can prove that

$$
\lim _{c \rightarrow \infty} \limsup _{n \rightarrow \infty} \mathbb{P}\left(\widehat{F}_{n}(x) \neq \widehat{F}_{n, c}(x)\right)=0 .
$$

It can be shown then that the process $\left\{F_{n}(\eta)-F_{n}(x),|\eta-x| \leqslant 2 c n^{-1 / 3}\right\}$ is asymptotically uniformly close to $\left\{G_{n}(\eta)-G_{n}(x),|\eta-x| \leqslant 2 c n^{-1 / 3}\right\}$, provided $\varepsilon_{1}, \ldots, \varepsilon_{n}$ are defined on some rich enough probability space. The result then follows 
from Taylor's expansion of $F$ and Lemmas 2.1 and 2.2, since $f^{\prime}$ is uniformly continuous in a neighbourhood of $x$.

The reason why we choose to prove Theorem 4.1 under global regularity conditions is that Lemmas 4.1 and 4.2 are useful to prove our main result, Theorem 5.2 below, which describes asymptotic global behaviour of $\widehat{F}_{n}-F_{n}$. Stating Theorem 4.1 under local assumptions would make the paper cumbersome.

LEMMA 4.1. - Assume we are given the regression model (1.1), where $x_{i}=i / n$, the $\varepsilon_{i}$ 's are centered independent and identically distributed random variables and $f$ is a differentiable function with bounded derivative. Let $F_{n}$ be the empirical process defined by (1.2) and let $F$ be defined by $F(t)=\int_{0}^{t} f(s) d s, t \in[0,1]$. If the $\varepsilon_{i}$ 's are defined on some rich enough probability space and if $\mathbb{E}\left|\varepsilon_{1}\right|^{p}$ is finite for some $p>0$ then there exist some $C_{p}>0$ and some standard Brownian motion $W$ such that for all $u>0$,

$$
\mathbb{P}\left(\sup _{t \in[0,1]}\left|F_{n}(t)-F(t)-\frac{1}{\sqrt{n}} W(t)\right|>u\right) \leqslant C_{p} n^{1-p} u^{-p} .
$$

If $\sup _{t}|f(t)| \leqslant s_{0}$ and $\sup _{t}\left|f^{\prime}(t)\right| \leqslant s_{1}$, then one can choose $C_{p}$ that only depends on $p$, $s_{0}$ and $s_{1}$.

Proof. - We assume without loss of generality that $u / 2>\left(s_{0}+s_{1}\right) / n$ (in the case where $u / 2 \leqslant\left(s_{0}+s_{1}\right) / n$, it suffices to choose $C_{p}$ large enough so that the upper bound in (4.9) is greater than one and the inequality is trivial). Suppose that the $\varepsilon_{i}$ 's are defined on some rich enough probability space so that the Sakhanenko's construction holds. In that case, see [9], there exists some standard Brownian motion $W_{0}$ such that

$$
\mathbb{E}\left(\sup _{1 \leqslant k \leqslant n}\left|\sum_{i=1}^{k} \varepsilon_{i}-W_{0}(k)\right|^{p}\right) \leqslant n \mathbb{E}\left|\varepsilon_{1}\right|^{p} .
$$

In the sequel, $W$ denotes the standard Brownian motion defined by $W(t)=W_{0}(n t) / \sqrt{n}$ and $[n t]$ denotes the integer part of $n t$. Let $d$ and $u$ be positive numbers with $d \leqslant 1$. For every $t \in[0,1]$ let $\pi(t)$ denote that element of $\{0, d / 4,2 d / 4, \ldots,([4 / d]+1) d / 4\}$ which is closer to $t$. Then, $|\pi(t)-t| \leqslant d / 8$ and $|\pi(t)-s| \leqslant 9 d / 8$ for every $s$ with $|t-s| \leqslant d$. Therefore,

$$
\mathbb{P}\left(\sup _{t \in[0,1],|t-s| \leqslant d}|W(t)-W(s)|>u\right) \leqslant 2 \sum_{k=0}^{\left[\frac{4}{d}\right]+1} \mathbb{P}\left(\sup _{\left|s-\frac{k d}{4}\right| \leqslant \frac{9 d}{8}}\left|W\left(\frac{k d}{4}\right)-W(s)\right|>\frac{u}{2}\right) .
$$

From time homogeneity and scaling properties of Brownian motion,

$$
\mathbb{P}\left(\sup _{t \in[0,1],|t-s| \leqslant d}|W(t)-W(s)|>u\right) \leqslant 2\left(\frac{4}{d}+2\right) \mathbb{P}\left(\sup _{|s| \leqslant 1}|W(s)|>\frac{u}{2 \sqrt{9 d / 8}}\right) .
$$

It thus follows from symmetry of Brownian motion and exponential inequality that

$$
\mathbb{P}\left(\sup _{t \in[0,1],|t-s| \leqslant d}|W(t)-W(s)|>u\right) \leqslant \frac{48}{d} \exp \left(-\frac{u^{2}}{9 d}\right)
$$


for all $u>0$ and $d \in(0,1]$. In particular, there exist some positive $A$ and $A^{\prime}$ such that for all positive $u$

$$
\mathbb{P}\left(\sup _{t \in[0,1]}\left|\frac{1}{\sqrt{n}} W\left(\frac{[n t]}{n}\right)-\frac{1}{\sqrt{n}} W(t)\right|>u / 4\right) \leqslant A n \exp \left(-\frac{n^{2} u^{2}}{9 \times 4^{2}}\right) \leqslant A^{\prime} n^{1-p} u^{-p} .
$$

Moreover, $\sup _{t}\left|\sum_{i \leqslant n t} f\left(x_{i}\right)-n F(t)\right| \leqslant s_{0}+s_{1}$ and therefore, the probability in (4.9) is less than or equal to

$$
\mathbb{P}\left(\sup _{1 \leqslant k \leqslant n}\left|\frac{1}{n} \sum_{i \leqslant k} \varepsilon_{i}-\frac{1}{\sqrt{n}} W\left(\frac{k}{n}\right)\right|>u / 4\right)+A^{\prime} n^{1-p} u^{-p} .
$$

The result now follows from Markov's inequality and (4.10).

In the following lemma, it is stated that the least concave majorant $\widehat{G}_{n}$ of $G_{n}$ at a given point $x$ is essentially unchanged if the process $G_{n}$ is restricted to some interval with length $4 c n^{-1 / 3}$ (where $c>0$ ) and centered at $x$. This result has to be compared to Lemma 3.2, where an analogous property of the canonical process $\widehat{X}-X$ is stated.

LEMMA 4.2. - Let $f$ be a decreasing function defined on $[0,1]$ and $G_{n}$ be defined by (4.8). Let $\widehat{G}_{n}$ and $\widehat{G}_{n, c}^{(x)}$ be the least concave majorants of the processes $\left\{G_{n}(\eta), \eta \in\right.$ $[0,1]\}$ and $\left\{G_{n}(\eta), \eta \in\left[x-2 c n^{-1 / 3}, x+2 c n^{-1 / 3}\right] \cap[0,1]\right\}$ respectively, where $c>0$ and $x \in[0,1]$. If $f$ is twice differentiable with $\inf _{t}\left|f^{\prime}(t)\right| \geqslant \varepsilon$, $\sup _{t}\left|f^{\prime}(t)\right| \leqslant s_{1}$ and $\sup _{t}\left|f^{\prime \prime}(t)\right| \leqslant s_{2}$ for some positive $\varepsilon, s_{1}$ and $s_{2}$, then there exist some positive $K$ and $K^{\prime}$ that only depend on $\varepsilon, s_{1}$ and $s_{2}$ such that

$$
\mathbb{P}\left(\widehat{G}_{n}(x) \neq \widehat{G}_{n, c}^{(x)}(x)\right) \leqslant 12 \exp \left(-K c^{3}\right)
$$

whenever $n \geqslant K^{\prime} c^{3}$.

Proof. - Fix $x \in[0,1]$. Let $\tilde{x}_{i}$ be the smallest real number $u \geqslant\left(x-2 c n^{-1 / 3}\right) \vee 0$ that satisfies $\widehat{G}_{n}(u)=\widehat{G}_{n, c}^{(x)}(u)$ (with the convention that the infimum of an empty set is $\left.\left(x+2 c n^{-1 / 3}\right) \wedge 1\right)$. If $\tilde{x}_{i}>x$ then by definition, $\widehat{G}_{n}(u) \neq \widehat{G}_{n, c}^{(x)}(u)$ for all $u \leqslant x$. In that case, there exist some $y \leqslant x-2 c n^{-1 / 3}, z \geqslant x$ such that the line segment joining $\left(y, G_{n}(y)\right)$ and $\left(z, G_{n}(z)\right)$ is above $\left(t, G_{n}(t)\right)$ for all $t \in(y, z)$. More specifically, this line segment is above $\left(x-c n^{-1 / 3}, G_{n}\left(x-c n^{-1 / 3}\right)\right)$, which implies that the slope of the line segment joining $\left(y, G_{n}(y)\right)$ and $\left(x-c n^{-1 / 3}, G_{n}\left(x-c n^{-1 / 3}\right)\right)$ is smaller than the slope of the line segment joining $\left(z, G_{n}(z)\right)$ and $\left(x-c n^{-1 / 3}, G_{n}\left(x-c n^{-1 / 3}\right)\right)$. We thus have $\tilde{x}_{i} \leqslant x$ whenever the following inequality holds for all $y \leqslant x-2 c n^{-1 / 3}$ and all $z \geqslant x$ :

$$
\frac{G_{n}(y)-G_{n}\left(x-c n^{-1 / 3}\right)}{y-x+c n^{-1 / 3}} \geqslant \frac{G_{n}(z)-G_{n}\left(x-c n^{-1 / 3}\right)}{z-x+c n^{-1 / 3}} .
$$

But the latter inequality holds whenever the left hand term is greater than some given real number $\alpha$ and the right hand term is smaller than $\alpha$. Therefore for every $\alpha>0$, $\mathbb{P}\left(\tilde{x}_{i}>x\right) \leqslant \mathbb{P}_{1}+\mathbb{P}_{2}$, where

$$
\mathbb{P}_{1}=\mathbb{P}\left(\exists y \leqslant x-2 c n^{-1 / 3}, G_{n}(y)-G_{n}\left(x-c n^{-1 / 3}\right)>\left(y-x+c n^{-1 / 3}\right) \alpha\right)
$$


and

$$
\mathbb{P}_{2}=\mathbb{P}\left(\exists z \geqslant x, G_{n}(z)-G_{n}\left(x-c n^{-1 / 3}\right)>\left(z-x+c n^{-1 / 3}\right) \alpha\right) .
$$

First we estimate the probability $\mathbb{P}_{1}$. For every real number $t$, we have $\mathbb{P}_{1} \leqslant \mathbb{P}_{1,1}+\mathbb{P}_{1,2}$, where

$$
\mathbb{P}_{1,1}=\mathbb{P}\left(G_{n}(x)-G_{n}\left(x-c n^{-1 / 3}\right)-c \alpha n^{-1 / 3}>t\right)
$$

and

$$
\mathbb{P}_{1,2}=\mathbb{P}\left(\exists y \leqslant x-2 c n^{-1 / 3}, G_{n}(x)-G_{n}(y)+(y-x) \alpha<t\right) .
$$

In the sequel, we fix $t=n^{-2 / 3} c^{2} f^{\prime}(x) / 4$ and $\alpha=f(x)+c n^{-1 / 3}\left|f^{\prime}(x)\right|$. Since $n^{-1 / 6} c^{1 / 2} W(1)$ is identical in law to $W(x)-W\left(x-c n^{-1 / 3}\right)$, we have

$$
\mathbb{P}_{1,1} \leqslant \mathbb{P}\left(\sqrt{c} W(1)>c \alpha n^{1 / 3}+n^{2 / 3} t+n^{2 / 3}\left(F\left(x-c n^{-1 / 3}\right)-F(x)\right)\right) .
$$

From Taylor's expansion,

$$
F\left(x-c n^{-1 / 3}\right)-F(x) \geqslant-c n^{-1 / 3} f(x)+\frac{c^{2}}{2} n^{-2 / 3} f^{\prime}(x)-\frac{c^{3}}{6} n^{-1} s_{2}
$$

since by definition, $\sup _{t}\left|f^{\prime \prime}(t)\right| \leqslant s_{2}$. Therefore,

$$
\mathbb{P}_{1,1} \leqslant \mathbb{P}\left(\sqrt{c} W(1)>\frac{c^{2}}{4}\left|f^{\prime}(x)\right|-\frac{s_{2}}{6} c^{3} n^{-1 / 3}\right) .
$$

If $3 n^{1 / 3} \varepsilon \geqslant 4 s_{2} c$, then $\mathbb{P}_{1,1}$ is no more than $\mathbb{P}\left(8 W(1)>c^{3 / 2} \varepsilon\right)$, which is, by exponential inequality, no more than $\exp \left(-K c^{3}\right)$ for some positive real number $K$ that only depends on $\varepsilon$. Let us estimate $\mathbb{P}_{1,2}$. By scaling and time homogeneity of Brownian motion, change of variable $z=n^{1 / 3}(x-y) / 2 c$ yields:

$\mathbb{P}_{1,2} \leqslant \mathbb{P}\left(\sup _{z \geqslant 1}\left\{n^{2 / 3}\left(F\left(x-2 c n^{-1 / 3} z\right)-F(x)\right)+\sqrt{2 c} W(z)+2 c \alpha n^{1 / 3} z\right\}>\frac{c^{2}\left|f^{\prime}(x)\right|}{4}\right)$.

Let $a$ be some real number with $a \varepsilon \geqslant 2 s_{1}$ (which implies $a \geqslant 1$ ). On the one hand, the first derivative of $f$ is non-positive. So by Taylor's expansion, for all $z \geqslant a$,

$$
n^{2 / 3}\left(F\left(x-2 c n^{-1 / 3} z\right)-F(x)\right)+2 c \alpha n^{1 / 3} z \leqslant-c^{2} z^{2} \varepsilon .
$$

It then follows that

$$
\begin{aligned}
& \mathbb{P}\left(\sup _{z \geqslant a}\left\{n^{2 / 3}\left(F\left(x-2 c n^{-1 / 3} z\right)-F(x)\right)+\sqrt{2 c} W(z)+2 c \alpha n^{1 / 3} z\right\}>\frac{c^{2}\left|f^{\prime}(x)\right|}{4}\right) \\
& \quad \leqslant \mathbb{P}\left(\sup _{z \geqslant a}\left\{W(z)-\frac{\varepsilon c^{3 / 2}}{\sqrt{2}} z^{2}\right\}>0\right),
\end{aligned}
$$

where by (3.3) the latter probability is no more than $\exp \left(-K c^{3}\right)$ for some $K>0$ that only depends on $\varepsilon$ and $a$. On the other hand, for all $z \in[1, a]$ there exists some $\xi_{z} \in[0,1]$ 
such that

$n^{2 / 3}\left(F\left(x-2 c n^{-1 / 3} z\right)-F(x)\right)+2 c \alpha n^{1 / 3} z=2 c^{2}\left|f^{\prime}(x)\right| z(1-z)-\frac{4}{3} c^{3} n^{-1 / 3} z^{3} f^{\prime \prime}\left(\xi_{z}\right)$,

where $2 c^{2}\left|f^{\prime}(x)\right| z(1-z) \leqslant 0$. Therefore there exists some $K^{\prime}>0$ that only depends on $\varepsilon, a$ and $s_{2}$ such that for all $z \in[1, a]$

$$
n^{2 / 3}\left(F\left(x-2 c n^{-1 / 3} z\right)-F(x)\right)+2 c \alpha n^{1 / 3} z+t n^{2 / 3} \leqslant-c^{2}\left|f^{\prime}(x)\right| / 8
$$

whenever $n \geqslant c^{3} K^{\prime}$. So there exists some $K>0$ that only depends on $\varepsilon$ and $a$ such that

$$
\begin{aligned}
& \mathbb{P}\left(\sup _{z \in[1, a]}\left\{n^{2 / 3}\left(F\left(x-2 c n^{-1 / 3} z\right)-F(x)\right)+\sqrt{2 c} W(z)+2 c \alpha n^{1 / 3} z\right\}>\frac{c^{2}\left|f^{\prime}(x)\right|}{4}\right) \\
& \quad \leqslant \exp \left(-K c^{3}\right)
\end{aligned}
$$

whenever $n \geqslant c^{3} K^{\prime}$. We thus have $\mathbb{P}_{1,2} \leqslant 2 \exp \left(-K c^{3}\right)$ whenever $n \geqslant c^{3} K^{\prime}$. Therefore $\mathbb{P}_{1} \leqslant 3 \exp \left(-K c^{3}\right)$ whenever $n \geqslant c^{3} K^{\prime}$. By using the same arguments, one can prove that there exist some positive real numbers $K$ and $K^{\prime}$ such that $\mathbb{P}_{2} \leqslant 3 \exp \left(-K c^{3}\right)$ whenever $n \geqslant c^{3} K^{\prime}$. Since $\mathbb{P}\left(\tilde{x}_{i}>x\right) \leqslant \mathbb{P}_{1}+\mathbb{P}_{2}$, it follows that $\mathbb{P}\left(\tilde{x}_{i}>x\right) \leqslant 6 \exp \left(-K c^{3}\right)$. Let $\tilde{x}_{s}$ be the greatest $u \leqslant\left(x+2 c n^{-1 / 3}\right) \wedge 1$ such that $\widehat{G}_{n}(u)=\widehat{G}_{n, c}^{(x)}(u)$, with the convention that the supremum of an empty set is $\left(x-2 c n^{-1 / 3}\right) \vee 0$. One can prove by using the same arguments as above that there exist some positive real numbers $K$ and $K^{\prime}$ that only depend on $\varepsilon, s_{1}$ and $s_{2}$ such that $\mathbb{P}\left(\tilde{x}_{s}<x\right) \leqslant 6 \exp \left(-K c^{3}\right)$ whenever $n \geqslant c^{3} K^{\prime}$. But $\widehat{G}_{n}(x)=\widehat{G}_{n, c}^{(x)}(x)$ whenever $\tilde{x}_{i} \leqslant x \leqslant \tilde{x}_{s}$, so the proof of Lemma 4.2 is complete.

We turn now to the proof of the main result of this section.

Proof of Theorem 4.1. - In the sequel, $F(t)$ denotes $\int_{0}^{t} f(s) d s, t \in[0,1]$. We assume without loss of generality that $\sigma=1$ and that the $\varepsilon_{i}$ 's are defined on some rich enough probability space so that the Sakhanenko's construction holds.

Let $G_{n}$ be the process defined by (4.8) where $W$ is some Brownian motion that satisfies (4.9) for some $p>3$. Then $n^{2 / 3} \sup _{t \in[0,1]}\left|F_{n}(t)-G_{n}(t)\right|$ converges to zero in probability as $n$ goes to infinity. If $\widehat{G}_{n}$ denotes the least concave majorant of the process $G_{n}$, then by Lemma $2.2, n^{2 / 3}\left(\widehat{F}_{n}(x)-\widehat{G}_{n}(x)\right)$ also converges to zero in probability as $n$ goes to infinity. By Lemmas 3.2 and 4.2 it thus suffices to prove that there exists some $k>0$ such that for all $c>0$,

$$
n^{2 / 3}\left(\widehat{G}_{n, c}^{(x)}(x)-G_{n}(x)\right) \stackrel{\mathcal{D}}{\longrightarrow}\left|\frac{f^{\prime}(x)}{2}\right|^{-1 / 3} \widehat{X}_{k c}^{(0)}(0), \quad \text { as } n \rightarrow \infty
$$

where $\widehat{G}_{n, c}^{(x)}$ and $\widehat{X}_{k c}^{(0)}$ are defined as in Lemmas 4.2 and 3.2. Fix $c>0$ and assume $n$ large enough so that $\left[x-2 c n^{-1 / 3}, x+2 c n^{-1 / 3}\right] \subset[0,1]$. By Lemma 2.1, $\left(\widehat{G}_{n, c}^{(x)}(x)-G_{n}(x)\right)$ is the least concave majorant at $\eta=0$ of the process

$$
\left\{G_{n}(x+\eta)-G_{n}(x), \eta \in\left[-2 c n^{-1 / 3}, 2 c n^{-1 / 3}\right]\right\} .
$$


By Taylor's expansion and time homogeneity of Brownian motion, it is identical in distribution to the concave majorant at $\eta=0$ of

$$
\left\{\eta f(x)+\frac{\eta^{2}}{2} f^{\prime}(x)+\frac{\eta^{3}}{6} f^{\prime \prime}\left(\xi_{\eta}\right)+\frac{1}{\sqrt{n}} W(\eta), \eta \in\left[-2 c n^{-1 / 3}, 2 c n^{-1 / 3}\right]\right\}
$$

where $\xi_{\eta} \in[0,1]$. Change of variable $\delta=n^{1 / 3}\left|f^{\prime}(x) / 2\right|^{2 / 3} \eta$, the three properties in Lemma 2.1 and scaling property of Brownian motion yield that $n^{2 / 3}\left|f^{\prime}(x) / 2\right|^{1 / 3} \times$ $\left(\widehat{G}_{n, c}^{(x)}(x)-G_{n}(x)\right)$ is identical in distribution to the least concave majorant at $\delta=0$ of

$$
\left\{-\delta^{2}+n^{-1 / 3} \frac{\delta^{3}}{6}\left|\frac{f^{\prime}(x)}{2}\right|^{-5 / 3} f^{\prime \prime}\left(\xi_{\delta}^{\prime}\right)+W(\delta), \delta \in\left[-2^{1 / 3}\left|f^{\prime}(x)\right|^{2 / 3} c, 2^{1 / 3}\left|f^{\prime}(x)\right|^{2 / 3} c\right]\right\}
$$

where $\xi_{\delta}^{\prime} \in[0,1]$. Since $f^{\prime \prime}$ is bounded and $\left|f^{\prime}\right|$ is bounded away from zero, it converges in distribution to the least concave majorant of

$$
\left\{W(\eta)-\eta^{2}, \eta \in\left[-2^{1 / 3}\left|f^{\prime}(x)\right|^{2 / 3} c, 2^{1 / 3}\left|f^{\prime}(x)\right|^{2 / 3} c\right]\right\}
$$

as $n$ goes to infinity. This proves (4.14) and completes the proof of the theorem.

\section{Global behaviour of $\widehat{\boldsymbol{F}}_{\boldsymbol{n}}-\boldsymbol{F}_{\boldsymbol{n}}$}

In this section we describe the asymptotic distribution of the $\mathbb{L}_{q}$-distance between $\widehat{F}_{n}$ and $F_{n}$ and provide the order of magnitude of the supremum distance between these processes.

\subsection{Statement of the main results}

We first state that the supremum distance between $\widehat{F}_{n}$ and $F_{n}$ is of order $((\log n) / n)^{2 / 3}$. Let $\varepsilon, s_{0}, s_{1}$ and $s_{2}$ be positive numbers and let $\mathcal{F}$ be the class of decreasing functions $f$ defined on $[0,1]$ that satisfy the following properties: $f$ is twice differentiable, $\inf _{t}\left|f^{\prime}(t)\right| \geqslant \varepsilon$ and $\sup _{t}\left|f^{(i)}(t)\right| \leqslant s_{i}$, where $f^{(i)}$ denotes the $i$ th derivative of $f$, $i=0,1,2$.

THEOREM 5.1. - Assume we are given the regression model (1.1), where $x_{i}=i / n$, the $\varepsilon_{i}$ 's are centered independent and identically distributed random variables with $\mathbb{E}\left|\varepsilon_{1}\right|^{3}<\infty$. Let $F_{n}$ be the empirical process defined by (1.2) and let $\widehat{F}_{n}$ be the least concave majorant of $F_{n}$. If $\mathcal{F}$ is not empty, then there exist some positive $C$ and $C^{\prime}$ such that for all $n$

$$
C \leqslant\left(\frac{n}{\log n}\right)^{2 / 3} \sup _{f \in \mathcal{F}} \mathbb{E}_{f}\left(\sup _{t \in[0,1]}\left|\widehat{F}_{n}(t)-F_{n}(t)\right|\right) \leqslant C^{\prime},
$$

where $\mathbb{E}_{f}$ denotes expectation in model (1.1). 
This result has to be compared with Theorem 1 of Kiefer and Wolfowitz [5]. Let $X_{1}, \ldots, X_{n}$ be independent and identically distributed random variables whose underlying density is assumed to be decreasing. In their Theorem 1, Kiefer and Wolfowitz state that the supremum distance between the empirical distribution function and its least concave majorant is $\mathcal{O}_{\text {a.s. }}\left(n^{-2 / 3} \log n\right)$. In fact, slight modification of their proof leads to the fact that this supremum distance is $\mathcal{O}_{\mathbb{P}}\left(((\log n) / n)^{2 / 3}\right)$. It is thus not surprising that, in our framework of regression estimation, the supremum distance between the empirical process and its least concave majorant is $\mathcal{O}_{\mathbb{P}}\left(((\log n) / n)^{2 / 3}\right)$. In our Theorem 5.1, we also provide a lower bound that proves that the order of magnitude of this distance is exactly $((\log n) / n)^{2 / 3}$.

We turn now to the statement of our main result: the asymptotic distribution of the $\mathbb{L}_{q}$-distance between $\widehat{F}_{n}$ and $F_{n}$ is given in the following theorem. Let us recall that the constants $m_{q}$ and $k_{q}$ defined by (3.4) exist and are finite (see Lemma 3.3).

THEOREM 5.2. - Assume we are given the regression model (1.1), where $x_{i}=i / n$, the $\varepsilon_{i}$ 's are centered independent and identically distributed random variables and $f$ is a decreasing twice differentiable function with bounded second derivative. Let $F_{n}$ be the empirical process defined by (1.2) and let $\widehat{F}_{n}$ be the least concave majorant of $F_{n}$. If $\inf _{t \in[0,1]}\left|f^{\prime}(t)\right|>0$ and if there exists some $p>6$ for which $\mathbb{E}\left|\varepsilon_{1}\right|^{p}$ is finite, then for every $q>1 / 2+3 /(2(p-3))$,

$$
n^{1 / 6}\left(n^{2 q / 3} \int_{0}^{1}\left|\widehat{F}_{n}(t)-F_{n}(t)\right|^{q} d t-m_{q} \int_{0}^{1}\left|\frac{f^{\prime}(t)}{2 \sigma^{4}}\right|^{-q / 3} d t\right) \stackrel{\mathcal{D}}{\longrightarrow} \mathcal{N}\left(0, \Gamma_{q}^{2}\right) \quad \text { as } n \rightarrow \infty,
$$

where

$$
\sigma^{2}=\mathbb{E}\left(\varepsilon_{1}^{2}\right), \quad \Gamma_{q}^{2}=2 k_{q} \sigma^{2 q} \int_{0}^{1}\left|f^{\prime}(t) / 2\right|^{-2(q+1) / 3} d t
$$

and where $m_{q}$ and $k_{q}$ are defined by (3.4).

\subsection{Proofs}

This section is devoted to the proof of Theorems 5.1 and 5.2. We assume here that $\sigma=1$ and that the $\varepsilon_{i}$ 's are defined on some rich enough probability space so that Lemma 4.1 holds. By Lemma 4.1, the empirical function $F_{n}$ can be uniformly approximated in distribution by the process $G_{n}$ defined by (4.8). Therefore, $\widehat{F}_{n}$ can be uniformly approximated by the least concave majorant $\widehat{G}_{n}$ of $G_{n}$, which can be itself approximated by the least concave majorant $\widehat{G}_{n, c}^{(x)}$ of $G_{n}$ restricted to a well chosen bounded interval (see Lemma 4.2). In order to obtain global results, we first state that this approximation can be performed uniformly in $x$ : it is stated in Lemma 5.1 that $\sup _{x}\left|\widehat{G}_{n}(x)-\widehat{G}_{n, c}^{(x)}(x)\right|$ is small whenever $c$ is large enough.

LEMMA 5.1. - Let $f$ be a decreasing function defined on $[0,1]$ and $G_{n}$ be defined by (4.8). Let $\widehat{G}_{n}$ and $\widehat{G}_{n, c}^{(x)}$ be the least concave majorants of the processes $\left\{G_{n}(\eta), \eta \in\right.$ $[0,1]\}$ and $\left\{G_{n}(\eta), \eta \in\left[x-2 c n^{-1 / 3}, x+2 c n^{-1 / 3}\right] \cap[0,1]\right\}$ respectively, where $c>0$ 
and $x \in[0,1]$. If $f$ is twice differentiable with $\inf _{t}\left|f^{\prime}(t)\right| \geqslant \varepsilon$, $\sup _{t}\left|f^{\prime}(t)\right| \leqslant s_{1}$ and $\sup _{t}\left|f^{\prime \prime}(t)\right| \leqslant s_{2}$ then there exist some positive $K$ and $K^{\prime}$ that only depend on $\varepsilon$, $s_{1}$ and $s_{2}$ such that for all $q>0$

$$
\mathbb{E}\left(\sup _{x \in[0,1]}\left|\widehat{G}_{n}(x)-\widehat{G}_{n, c}^{(x)}(x)\right|^{q}\right) \leqslant A_{q} n^{\frac{1}{3}-\frac{q}{2}} c^{-1} \exp \left(-K c^{3}\right),
$$

for some $A_{q}>0$ that only depends on $\varepsilon, s_{1}$ and $s_{2}$ and whenever $n \geqslant K^{\prime} c^{3}$.

Proof. - The development of the proof is similar to the one of Lemma 4.2. We use here the notation $\tilde{x}_{i}$ defined in the proof of Lemma 4.2. For every $x \in[0,1]$, let $\alpha_{x}=f(x)+c n^{-1 / 3}\left|f^{\prime}(x)\right|$ and $t_{x}=n^{-2 / 3} c^{2} f^{\prime}(x) / 4$. We have $\mathbb{P}\left(\exists x \in[0,1], \quad\left(\tilde{x}_{i}-x\right)>\right.$ $0) \leqslant \mathbb{P}_{1}+\mathbb{P}_{2}$, where

$$
\begin{aligned}
\mathbb{P}_{1}=\mathbb{P} & \left(\exists x \in[0,1], \exists y \leqslant x-2 c n^{-1 / 3},\right. \\
& \left.G_{n}(y)-G_{n}\left(x-c n^{-1 / 3}\right)>\left(y-x+c n^{-1 / 3}\right) \alpha_{x}\right)
\end{aligned}
$$

and

$$
\mathbb{P}_{2}=\mathbb{P}\left(\exists x \in[0,1], \exists z \geqslant x, G_{n}(z)-G_{n}\left(x-c n^{-1 / 3}\right)>\left(z-x+c n^{-1 / 3}\right) \alpha_{x}\right) .
$$

Also, $\mathbb{P}_{1} \leqslant \mathbb{P}_{1,1}+\mathbb{P}_{1,2}$, where

$$
\mathbb{P}_{1,1}=\mathbb{P}\left(\exists x \in[0,1], G_{n}(x)-G_{n}\left(x-c n^{-1 / 3}\right)-c \alpha_{x} n^{-1 / 3}>t_{x}\right)
$$

and

$$
\mathbb{P}_{1,2}=\mathbb{P}\left(\exists x \in[0,1], \exists y \leqslant x-2 c n^{-1 / 3}, G_{n}(x)-G_{n}(y)+(y-x) \alpha_{x}<t_{x}\right) .
$$

We obtain by using Taylor's expansion

$$
\mathbb{P}_{1,1} \leqslant \mathbb{P}\left(\sup _{x \in[0,1]} n^{1 / 6}\left(W(x)-W\left(x-c n^{-1 / 3}\right)\right)>\frac{c^{2}}{8} \varepsilon\right),
$$

whenever $3 n^{1 / 3} \varepsilon \geqslant 4 s_{2} c$. Inequality (4.11) holds for every $u>0, d \in(0,1]$. There thus exist some positive numbers $A, K$ and $K^{\prime}$ such that $c \mathbb{P}_{1,1} \leqslant A n^{1 / 3} \exp \left(-K c^{3}\right)$ whenever $n \geqslant K^{\prime} c^{3}$. Let $a$ be some real number with $a \varepsilon \geqslant 2 s_{1}$. For all $x \in[0,1], z \geqslant 1$, let $Y_{n}(x, z)$ be defined by

$$
Y_{n}(x, z)=n^{2 / 3}\left(G_{n}\left(x-2 c n^{-1 / 3} z\right)-G_{n}(x)\right)+2 c \alpha_{x} n^{1 / 3} z+n^{2 / 3} t_{x} .
$$

We have (4.12) for all $x \in[0,1]$ and all $z \geqslant a$ so

$$
\begin{aligned}
& \mathbb{P}\left(\sup _{x, z}\left\{Y_{n}(x, z)\right\}>0\right) \\
& \quad \leqslant \mathbb{P}\left(\sup _{x, z}\left\{n^{1 / 6}\left(W\left(x-2 c n^{-1 / 3} z\right)-W(x)\right)-\varepsilon c^{2} z^{2}\right\}>\frac{c^{2} \varepsilon}{4}\right),
\end{aligned}
$$


where the supremum is taken over the set

$$
\left\{(x, z), x \in[0,1], z \in\left[a ; x n^{1 / 3} /(2 c)\right]\right\} .
$$

For all $n \in \mathbb{N}$, let $k_{n}$ be the integer part of $n^{1 / 3} c^{-1}$ and for all $j \in\left\{1, \ldots, k_{n}\right\}$, let $t_{j}=j / k_{n}$. From (3.3) there exists some $K>0$ that only depends on $\varepsilon$ and $a$ such that for all $j \in\left\{1, \ldots, k_{n}\right\}$,

$$
\mathbb{P}\left(\sup _{z \geqslant a}\left\{n^{1 / 6}\left(W\left(t_{j}-2 c n^{-1 / 3} z\right)-W\left(t_{j}\right)\right)-\varepsilon c^{2} z^{2}\right\}>0\right) \leqslant \exp \left(-K c^{3}\right) .
$$

By symmetry and time homogeneity,

$$
\begin{gathered}
\mathbb{P}\left(\sup _{x \in\left[t_{j-1}, t_{j}\right], y \in[0,1]}\left\{n^{1 / 6}\left(W(x-y)-W\left(t_{j}-y\right)\right)\right\}>\frac{c^{2} \varepsilon}{8}\right) \\
\leqslant \mathbb{P}\left(\sup _{y \in[0,1],|u-y| \leqslant 1 / k_{n}}|W(u)-W(y)|>\frac{c^{2} \varepsilon n^{-1 / 6}}{8}\right)
\end{gathered}
$$

for all $j \in\left\{1, \ldots, k_{n}\right\}$, where $t_{0}=0$. Assume $n^{1 / 3} c^{-1} \geqslant 2$. Then $k_{n} \geqslant n^{1 / 3} c^{-1} / 2 \geqslant 1$ and from (4.11) there exist some $A>0$ and $K>0$ such that the latter probability is less than or equal to $A k_{n} \exp \left(-K c^{3}\right)$. Therefore, there exist some $A, K$ and $K^{\prime}$ such that the probability in (5.15) is less than or equal to $A k_{n}^{2} \exp \left(-K c^{3}\right)$ whenever $n \geqslant K^{\prime} c^{3}$. From (4.11) and (4.13) there exist some positive $A, K$ and $K^{\prime}$ such that

$$
\mathbb{P}\left(\sup _{x \in[0,1], z \in[1, a]}\left\{Y_{n}(x, z)\right\}>0\right) \leqslant A n^{1 / 3} c^{-1} \exp \left(-K c^{3}\right)
$$

whenever $n \geqslant K^{\prime} c^{3}$. There thus exist some positive $A, K, K^{\prime}$ such that

$$
\mathbb{P}_{1,2} \leqslant A n^{2 / 3} c^{-2} \exp \left(-K c^{3}\right)
$$

whenever $n \geqslant K^{\prime} c^{3}$. It follows that $c^{2} \mathbb{P}_{1} \leqslant A n^{2 / 3} \exp \left(-K c^{3}\right)$ for some positive $A$ and $K$, whenever $n \geqslant K^{\prime} c^{3}$. Repeating the same kind of arguments, we obtain that there exist some $A, K$ and $K^{\prime}$ that only depend on $\varepsilon, s_{1}$ and $s_{2}$ such that

$$
\mathbb{P}\left(\exists x \in[0,1], \widehat{G}_{n}(x) \neq \widehat{G}_{n, c}^{(x)}(x)\right) \leqslant A n^{2 / 3} c^{-2} \exp \left(-K c^{3}\right)
$$

whenever $n \geqslant K^{\prime} c^{3}$. It is assumed that $F$ is concave so by Lemma 2.2,

$$
\sup _{x \in[0,1]}\left|\widehat{G}_{n}(x)-\widehat{G}_{n, c}^{(x)}(x)\right| \leqslant 2 \sup _{x \in[0,1]}\left|G_{n}(x)-F(x)\right| \leqslant \frac{2}{\sqrt{n}} \sup _{x \in[0,1]}|W(x)| .
$$

By Hölder's inequality we have

$$
\begin{aligned}
& \mathbb{E}\left(\sup _{x \in[0,1]}\left|\widehat{G}_{n}(x)-\widehat{G}_{n, c}^{(x)}(x)\right|^{q}\right) \\
& \quad \leqslant \frac{2^{q}}{n^{q / 2}} \mathbb{E}^{1 / 2}\left(\sup _{x \in[0,1]}|W(x)|^{2 q}\right) \mathbb{P}^{1 / 2}\left(\exists x \in[0,1], \widehat{G}_{n}(x) \neq \widehat{G}_{n, c}^{(x)}(x)\right) .
\end{aligned}
$$


This last inequality combined with (5.16) completes the proof of the lemma.

Proof of Theorem 5.1. - In the sequel, $\mathbb{P}_{f}$ denotes probability in the model (1.1). Let $G_{n}$ be the process defined by (4.8), where $W$ is some standard Brownian motion that satisfies (4.9) where $p=3$ and $C_{3}$ only depends on $s_{1}$ and $s_{0}$. Let $\widehat{G}_{n}$ be the least concave majorant of $\left\{G_{n}(t), t \in[0,1]\right\}$. By (4.9) and Fubini's Theorem we have

$$
\mathbb{E}_{f}\left(\sup _{t \in[0,1]}\left|F_{n}(t)-G_{n}(t)\right|\right) \leqslant \int_{0}^{\infty} 1 \wedge\left(C_{3} n^{-2} u^{-3}\right) d u .
$$

There thus exists some $C^{\prime}>0$ such that for all $f \in \mathcal{F}$ and all $n$,

$$
\mathbb{E}_{f}\left(\sup _{t \in[0,1]}\left|F_{n}(t)-G_{n}(t)\right|\right) \leqslant C^{\prime} .
$$

From Lemma 2.2 we also have

$$
n^{2 / 3} \mathbb{E}_{f}\left(\sup _{t \in[0,1]}\left|\widehat{F}_{n}(t)-\widehat{G}_{n}(t)\right|\right) \leqslant C^{\prime} .
$$

Let $c_{n}=\left(c_{0} \log n\right)^{1 / 3}$ for some large enough $c_{0}>0$ and let $d_{n}=\left(d_{0} \log n\right)^{1 / 3}$ for some $\left.d_{0} \in\right] 0,1 /\left(10 \varepsilon^{2}\right)\left[\right.$. We will prove that on the one hand, there exists some $C^{\prime}>0$ such that

$$
\left(\frac{n}{\log n}\right)^{2 / 3} \mathbb{E}_{f}\left(\sup _{t \in[0,1]}\left|\widehat{G}_{n, c_{n}}^{(t)}(t)-G_{n}(t)\right|\right) \leqslant C^{\prime}
$$

for all large enough $n$ and all $f \in \mathcal{F}$ and on the other hand, there exists some $C>0$ such that

$$
\left(\frac{n}{\log n}\right)^{2 / 3} \mathbb{E}_{f_{0}}\left(\sup _{t \in[0,1]}\left|\widehat{G}_{n, d_{n}}^{(t)}(t)-G_{n}(t)\right|\right) \geqslant C
$$

for some $f_{0} \in \mathcal{F}$ and all large enough $n$. From Lemma 5.1 and since for every $t$, $G_{n}(t) \leqslant \widehat{G}_{n, d_{n}}^{(t)}(t) \leqslant \widehat{G}_{n}(t)$, Theorem 5.1 follows from these last four inequalities. For all $t \in[0,1]$, let $\widehat{Z}_{c_{n}}(t)$ be the least concave majorant at time zero of the process

$$
\left\{-\eta^{2}+n^{1 / 6}\left|\frac{f^{\prime}(t)}{2}\right|^{1 / 3}\left(W\left(t+n^{-1 / 3}\left|f^{\prime}(t) / 2\right|^{-2 / 3} \eta\right)-W(t)\right), \eta \in I_{n}(t)\right\},
$$

where

$$
I_{n}(t)=\left[-\left|\frac{f^{\prime}(t)}{2}\right|^{2 / 3}\left(\left(n^{1 / 3} t\right) \wedge\left(2 c_{n}\right)\right) ;\left|\frac{f^{\prime}(t)}{2}\right|^{2 / 3}\left(\left(n^{1 / 3}(1-t)\right) \wedge\left(2 c_{n}\right)\right)\right] .
$$

The least concave majorant of a process at a given time is less than or equal to the supremum of this process so for all $x>0$ and all $f \in \mathcal{F}$,

$$
\mathbb{P}_{f}\left(\sup _{t \in[0,1]}\left|\widehat{Z}_{c_{n}}(t)\right|>x\right) \leqslant \mathbb{P}_{f}\left(n^{1 / 6}\left(s_{1} / 2\right)^{1 / 3} \sup _{t \in[0,1],|t-u| \leqslant 2 c_{n} n^{-1 / 3}}|W(u)-W(t)|>x\right) .
$$


From (4.11), there thus exist some positive $A$ and $K$ such that for all $x>0$ and all $f \in \mathcal{F}$,

$$
\mathbb{P}_{f}\left(\sup _{t \in[0,1]}\left|\widehat{Z}_{c_{n}}(t)\right|>x\right) \leqslant\left(A n^{1 / 3} c_{n}^{-1} \exp \left(-K x^{2} / c_{n}\right)\right) \wedge 1
$$

whenever $n$ is large enough. Therefore, there exists some $C^{\prime}>0$ such that for all $f \in \mathcal{F}$ and all large enough $n$,

$$
\mathbb{E}_{f}\left(\sup _{t \in[0,1]}\left|\widehat{Z}_{c_{n}}(t)\right|\right) \leqslant C^{\prime}(\log n)^{2 / 3} .
$$

For every $t \in[0,1]$ and every $\eta \in I_{n}(t)$, let $Z_{c_{n}}(t, \eta)$ denote the value of the process (5.19) at time $\eta$. It follows from Taylor's expansion and the three properties in Lemma 2.1 that $n^{2 / 3}\left|f^{\prime}(t) / 2\right|^{1 / 3}\left(\widehat{G}_{n, c_{n}}^{(t)}(t)-G_{n}(t)\right)$ is the least concave majorant at time zero of the process

$$
\left\{Z_{c_{n}}(t, \eta)+n^{-1 / 3} \frac{\eta^{3}}{6}\left|\frac{f^{\prime}(t)}{2}\right|^{-5 / 3} f^{\prime \prime}\left(\xi_{\eta}\right), \eta \in I_{n}(t)\right\},
$$

where for all $\eta, \xi_{\eta}$ is some number in $[0,1]$. By Lemma 2.2, there exists some $K>0$ that only depends on $s_{1}$ and $s_{2}$ such that

$$
\left.\sup _{t \in[0,1]}\left|\widehat{Z}_{c_{n}}(t)-n^{2 / 3}\right| \frac{f^{\prime}(t)}{2}\right|^{1 / 3}\left(\widehat{G}_{n, c_{n}}^{(t)}(t)-G_{n}(t)\right) \mid \leqslant K n^{-1 / 3} c_{n}^{3} .
$$

We obtain (5.17) by combining (5.20) and (5.21).

Let $f_{0}$ be the decreasing function defined for $t \in[0,1]$ by $f_{0}(t)=-\varepsilon t+s_{0}$. Then, $\sup _{t}\left|f_{0}(t)\right|=\max \left(s_{0}, \varepsilon-s_{0}\right)$. But for all $f \in \mathcal{F}, f(0)-f(1) \geqslant \varepsilon$ and therefore, $2 s_{0} \geqslant \varepsilon$. It follows that $\sup _{t}\left|f_{0}(t)\right|=s_{0}$ and $f_{0} \in \mathcal{F}$. Let $k_{n}$ denote the integer part of $n^{1 / 3}\left(4 d_{n}\right)^{-1}$ and for all $j \in\left\{1, \ldots, k_{n}\right\}$, let $t_{j}=j / k_{n}$. Moreover, let $x=d_{0}^{2 / 3}|\varepsilon / 2|^{4 / 3}$. By Markov's inequality

$$
\left(\frac{1}{\log n}\right)^{2 / 3} \mathbb{E}_{f_{0}}\left(\sup _{1 \leqslant j \leqslant k_{n}-1}\left|\widehat{Z}_{d_{n}}\left(t_{j}\right)\right|\right) \geqslant x \mathbb{P}_{f_{0}}\left(\sup _{1 \leqslant j \leqslant k_{n}-1}\left|\widehat{Z}_{d_{n}}\left(t_{j}\right)\right|>x(\log n)^{2 / 3}\right) .
$$

By (5.21) where $c_{n}$ stands for $d_{n}$, it suffices to prove that

$$
\liminf _{n \rightarrow \infty} \mathbb{P}_{f_{0}}\left(\sup _{1 \leqslant j \leqslant k_{n}-1}\left|\widehat{Z}_{d_{n}}\left(t_{j}\right)\right|>x(\log n)^{2 / 3}\right)>0
$$

in order to prove (5.18). The increments of Brownian motion are independent and therefore the random variables $\widehat{Z}_{d_{n}}\left(t_{j}\right), j \in\left\{1, \ldots, k_{n}\right\}$, are independent. Moreover, $n^{1 / 3} k_{n}^{-1} \geqslant 4 d_{n}$ so it follows from scaling and time homogeneity of Brownian motion that the random variable $\widehat{Z}_{d_{n}}\left(t_{j}\right)$ is identical in distribution to the least concave majorant at time zero of $\left\{-\eta^{2}+W(\eta),|\eta| \leqslant 2 d_{n}\left|f^{\prime}\left(t_{j}\right) / 2\right|^{2 / 3}\right\}$, for all $j \in\left\{1, \ldots, k_{n}-1\right\}$. Therefore when $f=f_{0}$, the random variables $\widehat{Z}_{d_{n}}\left(t_{j}\right), j \in\left\{1, \ldots, k_{n}-1\right\}$, are independent and 
identically distributed. It follows that

$$
\mathbb{P}_{f_{0}}\left(\sup _{1 \leqslant j \leqslant k_{n}-1}\left|\widehat{Z}_{d_{n}}\left(t_{j}\right)\right|>x(\log n)^{2 / 3}\right)=1-\mathbb{P}_{f_{0}}^{k_{n}-1}\left(\left|\widehat{Z}_{d_{n}}\left(t_{1}\right)\right| \leqslant x(\log n)^{2 / 3}\right),
$$

where $\widehat{Z}_{d_{n}}\left(t_{1}\right) \geqslant 0$. The least concave majorant of a process $X$ at time zero is greater than or equal to either the supremum of $\{X(\eta), \eta \leqslant 0\}$ or the supremum of $\{X(\eta), \eta \geqslant 0\}$. Moreover, $\{W(\eta), \eta \geqslant 0\}$ is independent of $\{W(\eta), \eta \leqslant 0\}$ and for any $b>0$, $\sup _{\eta \in[0, b]}\left\{-\eta^{2}+W(\eta)\right\}$ is identical in distribution to $\sup _{\eta \in[-b, 0]}\left\{-\eta^{2}+W(\eta)\right\}$. We fix $b_{n}=2 d_{n}|\varepsilon / 2|^{2 / 3}$. Then the right hand term in (5.23) is greater than or equal to

$$
1-\left(1-\mathbb{P}^{2}\left(\sup _{\eta \in\left[0, b_{n}\right]}\left\{-\eta^{2}+W(\eta)\right\}>x(\log n)^{2 / 3}\right)\right)^{k_{n}-1} .
$$

By definition, $x=d_{0}^{2 / 3}|\varepsilon / 2|^{4 / 3}$. We thus have

$\mathbb{P}\left(\sup _{\eta \in\left[0, b_{n}\right]}\left\{-\eta^{2}+W(\eta)\right\}>x(\log n)^{2 / 3}\right) \geqslant \mathbb{P}\left(\sup _{\eta \in\left[0, b_{n}\right]}\{W(\eta)\}>5(\log n)^{2 / 3}\left(\frac{\varepsilon^{2} d_{0}}{4}\right)^{2 / 3}\right)$

where $\sup _{\eta \in\left[0, b_{n}\right]}\{W(\eta)\}$ has the same distribution as $\sqrt{b_{n}}|W(1)|$. Let $\phi$ be the distribution function of $W(1)$. It is well known that $1-\phi(t) \sim \phi^{\prime}(t) / t$ as $t$ goes to infinity so there exists some $t_{0}$ such that $1-\phi(t) \geqslant \phi^{\prime}(t) / 2 t$ for all $t \geqslant t_{0}$. We thus obtain for large enough $n$

$$
\mathbb{P}_{f_{0}}\left(\sup _{0 \leqslant j \leqslant k_{n}-1}\left|\widehat{Z}_{d_{n}}\left(t_{j}\right)\right|>x(\log n)^{2 / 3}\right) \geqslant 1-\left(1-\frac{4}{25 \pi \varepsilon^{2} d_{0} \log n} n^{-\frac{25}{8} d_{0} \varepsilon^{2}}\right)^{k_{n}-1},
$$

that converges to one as $n$ goes to infinity since by assumption $d_{0}<1 /\left(10 \varepsilon^{2}\right)$. This proves (5.22) and completes the proof of the theorem.

Proof of Theorem 5.2. - Let $x$ and $y$ be positive numbers and fix $r \in[0,1]$. Since for all $t \in[0,1], 1-t^{r} \leqslant(1-t)^{r}$ we have

$$
\left|x^{r}-y^{r}\right| \leqslant|x-y|^{r} .
$$

Fix now $r \geqslant 1$. There exists some $z$ lying between $x$ and $y$ such that $x^{r}-y^{r}=$ $r(x-y) z^{r-1}$. Since $r \geqslant 1$ we obtain

$$
\left|x^{r}-y^{r}\right| \leqslant r|x-y|(x+y)^{r-1} .
$$

Let $p$ be some positive number with $p>6$ and $\mathbb{E}\left|\varepsilon_{1}\right|^{p}<\infty$. Fix $q>1 / 2+3 /(2(p-3))$. Let $G_{n}$ be the process defined by (4.8), where $W$ is some standard Brownian motion that satisfies (4.9). Then,

$$
\sup _{t \in[0,1]}\left|F_{n}(t)-G_{n}(t)\right|=\mathrm{O}_{\mathbb{P}}\left(n^{1 / p-1}\right),
$$


which implies

$$
\sup _{t \in[0,1]}\left|F_{n}(t)-G_{n}(t)\right|^{q}=\mathrm{o}_{\mathbb{P}}\left(n^{-1 / 6-2 q / 3}\right) .
$$

Let $\widehat{G}_{n}$ be the least concave majorant of $\left\{G_{n}(\eta), \eta \in[0,1]\right\}$. Assume first $q \leqslant 1$. By (5.24), Lemma 2.2 and the latter equality,

$$
\int_{0}^{1}|| G_{n}(t)-\left.\widehat{G}_{n}(t)\right|^{q}-\left|F_{n}(t)-\widehat{F}_{n}(t)\right|^{q} \mid d t=\mathrm{o}_{\mathbb{P}}\left(n^{-1 / 6-2 q / 3}\right) .
$$

Assume now $q>1$. From (5.25),

$$
\begin{aligned}
& \int_{0}^{1}|| G_{n}(t)-\left.\widehat{G}_{n}(t)\right|^{q}-\left|F_{n}(t)-\widehat{F}_{n}(t)\right|^{q} \mid d t \\
& \quad \leqslant 2 q \sup _{t \in[0,1]}\left|F_{n}(t)-G_{n}(t)\right| \int_{0}^{1}\left(2\left|G_{n}(t)-\widehat{G}_{n}(t)\right|+2\left|F_{n}(t)-G_{n}(t)\right|\right)^{q-1} d t .
\end{aligned}
$$

Let $c_{n}=\left(c_{0} \log n\right)^{1 / 3}$ for some large enough positive real number $c_{0}$ and for every $t \in[0,1]$ let $\widehat{Z}_{c_{n}}(t)$ be the least concave majorant at time zero of the process (5.19). For all $t, \widehat{Z}_{c_{n}}(t) \geqslant 0$ and $\widehat{Z}_{c_{n}}(t)$ is less than or equal to a random variable distributed as $\widehat{X}(0)$, the least concave majorant at time zero of $\left\{-\eta^{2}+W(\eta), \eta \in \mathbb{R}\right\}$. By Lemma 3.3 there thus exists some $C_{q}>0$ such that

$$
\sup _{t \in[0,1]} \mathbb{E}^{1 /(q-1)}\left|\widehat{Z}_{c_{n}}(t)\right|^{q-1} \leqslant C_{q} .
$$

By Lemma 5.1 and (5.21), there thus exist some positive $A_{q}$ and $K_{q}$ such that for every $t \in[0,1]$

$$
\begin{aligned}
& \mathbb{E}^{1 /(q-1)}\left|G_{n}(t)-\widehat{G}_{n}(t)\right|^{q-1} \\
& \quad \leqslant A_{q}\left[n^{-1} c_{n}^{3}+n^{-2 / 3}+n^{1 / 3(q-1)-1 / 2} c_{n}^{-1 /(q-1)} \exp \left(-K_{q} c_{n}^{3}\right)\right] .
\end{aligned}
$$

It then follows from (5.26) that

$$
\int_{0}^{1}\left(2\left|G_{n}(t)-\widehat{G}_{n}(t)\right|+2\left|F_{n}(t)-G_{n}(t)\right|\right)^{q-1} d t=\mathrm{O}_{\mathbb{P}}\left(n^{-2 q / 3+2 / 3}\right) .
$$

Therefore, since $p>6$, (5.27) still holds in the case where $q \geqslant 1$, which implies

$$
\int_{0}^{1}\left|F_{n}(t)-\widehat{F}_{n}(t)\right|^{q} d t=\int_{0}^{1}\left|G_{n}(t)-\widehat{G}_{n}(t)\right|^{q} d t+\mathrm{o}_{\mathbb{P}}\left(n^{-1 / 6-2 q / 3}\right)
$$


for every $q>1 / 2+3 /(2(p-3))$. One can prove in the same way that

$$
n^{2 q / 3} \int_{0}^{1}\left|G_{n}(t)-\widehat{G}_{n}(t)\right|^{q} d t=\int_{0}^{1}\left|\frac{f^{\prime}(t)}{2}\right|^{-q / 3}\left|\widehat{Z}_{c_{n}}(t)\right|^{q} d t+\mathrm{o}_{\mathbb{P}}\left(n^{-1 / 6}\right)
$$

for every $q>1 / 2+3 /(2(p-3))$. Theorem 5.2 then follows from the following lemma (recall $\sigma=1$ ).

LEMMA 5.2. - Let $W$ be some standard two-sided Brownian motion, let $f$ be a twice differentiable function on $[0,1]$ with bounded second derivative and $\inf _{t}\left|f^{\prime}(t)\right|>0$. Let $c_{n}=\left(c_{0} \log n\right)^{1 / 3}$ for some fixed positive real number $c_{0}$ and for every $t \in[0,1]$ and $n \in \mathbb{N}$, let $\widehat{Z}_{c_{n}}(t)$ be the least concave majorant at time zero of the process (5.19). If $c_{0}$ is large enough then for all $q>0$ on the one hand,

$$
\lim _{n \rightarrow \infty} n^{1 / 6}\left(\int_{0}^{1}\left(\mathbb{E}\left|\widehat{Z}_{c_{n}}(t)\right|^{q}-m_{q}\right)\left|\frac{f^{\prime}(t)}{2}\right|^{-q / 3} d t\right)=0
$$

and on the other hand

$$
n^{1 / 6}\left(\int_{0}^{1}\left|\frac{f^{\prime}(t)}{2}\right|^{-q / 3}\left(\left|\widehat{Z}_{c_{n}}(t)\right|^{q}-\mathbb{E}\left|\widehat{Z}_{c_{n}}(t)\right|^{q}\right) d t\right) \stackrel{\mathcal{D}}{\longrightarrow} \mathcal{N}\left(0, \Gamma_{q}^{2}\right) \text { as } n \rightarrow \infty
$$

where $\Gamma_{q}^{2}=2 k_{q} \int_{0}^{1}\left|f^{\prime}(t) / 2\right|^{-2(q+1) / 3} d t$ and where $m_{q}$ and $k_{q}$ are defined by (3.4).

Proof. - Fix $q>0$. Let $\widehat{X}(0)$ be the least concave majorant at time zero of $\left\{-\eta^{2}+\right.$ $W(\eta), \eta \in \mathbb{R}\}$ and for every $d>0$, let $\widehat{X}_{d}(0)$ be the least concave majorant at time zero of $\left\{-\eta^{2}+W(\eta), \quad|\eta| \leqslant 2 d\right\}$. It follows from scaling and time homogeneity of Brownian motion that for all $t \in\left[2 c_{n} n^{-1 / 3}, 1-2 c_{n} n^{-1 / 3}\right], \widehat{Z}_{c_{n}}(t)$ is identical in distribution to $\widehat{X}_{d_{n}(t)}(0)$, where $d_{n}(t)=c_{n}\left|f^{\prime}(t) / 2\right|^{2 / 3}$. Moreover, for all $t \in[0,1]$, $\widehat{Z}_{c_{n}}(t) \geqslant 0$ and $\widehat{Z}_{c_{n}}(t)$ is less than or equal to a random variable distributed as $\widehat{X}(0)$. From Lemma 3.3, $\sup _{t \in[0,1]} \mathbb{E}\left|\widehat{Z}_{c_{n}}(t)\right|^{q}$ is thus finite. Also, $\sup _{t \in[0,1]} \mathbb{E}\left|\widehat{X}_{d_{n}}(0)\right|^{q}$ is finite since $0 \leqslant \widehat{X}_{d_{n}(t)}(0) \leqslant \widehat{X}(0)$. Therefore,

$$
\int_{0}^{1} \mathbb{E}\left|\widehat{Z}_{c_{n}}(t)\right|^{q}\left|\frac{f^{\prime}(t)}{2}\right|^{-q / 3} d t=\int_{0}^{1} \mathbb{E}\left|\widehat{X}_{d_{n}(t)}(0)\right|^{q}\left|\frac{f^{\prime}(t)}{2}\right|^{-q / 3} d t+\mathrm{O}\left(c_{n} n^{-1 / 3}\right) .
$$

Since $0 \leqslant \widehat{X}_{d_{n}(t)}(0) \leqslant \widehat{X}(0)$ we have

$$
\left.|\mathbb{E}| \widehat{X}_{d_{n}(t)}(0)\right|^{q}-\mathbb{E}|\widehat{X}(0)|^{q} \mid \leqslant \mathbb{E}\left(|\widehat{X}(0)|^{q} \mathbb{1}_{\widehat{X}_{d_{n}(t)}(0) \neq \widehat{X}(0)}\right) .
$$

Let $\varepsilon$ be some positive real number with $\inf _{t}\left|f^{\prime}(t)\right| \geqslant \varepsilon$. By Lemma 3.3, $\mathbb{E}|\widehat{X}(0)|^{p}$ is finite for all $p>0$. By Hölder's inequality and Lemma 3.2 there thus exists some $A_{q}>0$ 
such that

$$
\left.|\mathbb{E}| \widehat{X}_{d_{n}(t)}(0)\right|^{q}-\mathbb{E}|\widehat{X}(0)|^{q} \mid \leqslant A_{q} \exp \left(-d_{n}(t)^{3} / 4\right) \leqslant A_{q} n^{-c_{0} \varepsilon^{2} / 16}
$$

If $c_{0}$ is large enough (that is $\left.c_{0}>8 /\left(3 \varepsilon^{2}\right)\right)$ then

$$
\lim _{n \rightarrow \infty} n^{1 / 6} \int_{0}^{1}\left(\mathbb{E}\left|\widehat{X}_{d_{n}(t)}(0)\right|^{q}-\mathbb{E}|\widehat{X}(0)|^{q}\right)\left|\frac{f^{\prime}(t)}{2}\right|^{-q / 3} d t=0 .
$$

So we obtain (5.28) and it remains to prove (5.29). In the sequel, $Y_{n}(t)$ denotes the random variable

$$
Y_{n}(t)=\left|\frac{f^{\prime}(t)}{2}\right|^{-q / 3}\left(\left|\widehat{Z}_{c_{n}}(t)\right|^{q}-\mathbb{E}\left|\widehat{Z}_{c_{n}}(t)\right|^{q}\right) .
$$

By Fubini's Theorem,

$$
\operatorname{var}\left(\int_{0}^{1} Y_{n}(t) d t\right)=2 \int_{0}^{1} \int_{s}^{1}\left|\frac{f^{\prime}(t) f^{\prime}(s)}{4}\right|^{-q / 3} \operatorname{cov}\left(\left|\widehat{Z}_{c_{n}}(t)\right|^{q},\left|\widehat{Z}_{c_{n}}(s)\right|^{q}\right) d t d s .
$$

For all $t, \widehat{Z}_{c_{n}}(t)$ only depends on the increments of $W$ between times $t-2 c_{n} n^{-1 / 3}$ and $t+2 c_{n} n^{-1 / 3}$. The increments of Brownian motion are independent so the random variables $\widehat{Z}_{c_{n}}(t)$ and $\widehat{Z}_{c_{n}}(s)$ are independent whenever $|t-s| \geqslant 4 c_{n} n^{-1 / 3}$. Moreover,

$$
\sup _{t, s}\left|\frac{f^{\prime}(t) f^{\prime}(s)}{4}\right|^{-q / 3}\left|\operatorname{cov}\left(\left|\widehat{Z}_{c_{n}}(t)\right|^{q},\left|\widehat{Z}_{c_{n}}(s)\right|^{q}\right)\right|<\infty .
$$

Change of variable thus yields

$$
\begin{aligned}
n^{1 / 3} \operatorname{var}\left(\int_{0}^{1} Y_{n}(t) d t\right)= & 0(1)+2 \int_{n^{-1 / 4}}^{1-n^{-1 / 4}} \int_{0}^{4 c_{n}}\left|\frac{f^{\prime}(s) f^{\prime}\left(s+n^{-1 / 3} t\right)}{4}\right|^{-q / 3} \\
& \times \operatorname{cov}\left(\left|\widehat{Z}_{c_{n}}(s)\right|^{q},\left|\widehat{Z}_{c_{n}}\left(s+n^{-1 / 3} t\right)\right|^{q}\right) d t d s .
\end{aligned}
$$

In the sequel, we assume $n$ large enough so that $n^{-1 / 4} \geqslant 2 c_{n} n^{-1 / 3}$. Fix $s \in\left[n^{-1 / 4}, 1-\right.$ $\left.n^{-1 / 4}\right]$ and for every $t \in \mathbb{R}$, let $W_{s}(t)=n^{1 / 6}\left[W\left(s+n^{-1 / 3} t\right)-W(s)\right]$. Then $W_{s}$ is a standard Brownian motion and by change of variable, $\widehat{Z}_{c_{n}}(s)$ is the least concave majorant at time zero of the process

$$
\left\{-\left|\frac{f^{\prime}(s)}{2}\right|^{4 / 3} \eta^{2}+\left|\frac{f^{\prime}(s)}{2}\right|^{1 / 3} W_{s}(\eta),|\eta| \leqslant 2 c_{n}\right\}
$$

Moreover, for all $t \in\left[0,4 c_{n}\right], \widehat{Z}_{c_{n}}\left(s+n^{-1 / 3} t\right)$ is the least concave majorant at time zero of the process

$$
\left\{-\left|\frac{f^{\prime}\left(s+n^{-1 / 3} t\right)}{2}\right|^{4 / 3} \eta^{2}+\left|\frac{f^{\prime}\left(s+n^{-1 / 3} t\right)}{2}\right|^{1 / 3}\left(W_{s}(\eta+t)-W_{s}(t)\right),|\eta| \leqslant 2 c_{n}\right\} .
$$


For every $\eta \in \mathbb{R}$, let $X(\eta)=-\eta^{2}+\tilde{W}_{s}(\eta)$, where $\tilde{W}_{s}$ is the Brownian motion defined by $\tilde{W}_{s}(\eta)=\left|f^{\prime}(s) / 2\right|^{1 / 3} W_{s}\left(\left|f^{\prime}(s) / 2\right|^{-2 / 3} \eta\right)$. Let $d_{n}=c_{n}\left|f^{\prime}(s) / 2\right|^{2 / 3}$ and for every $u \in \mathbb{R}$, let $\widehat{X}_{d_{n}}(u)$ be the least concave majorant at time $u$ of

$$
\left\{-\eta^{2}+\tilde{W}_{s}(\eta),|\eta-u| \leqslant 2 d_{n}\right\}
$$

Let denote $\widehat{X}_{d_{n}}\left(\left|f^{\prime}(s) / 2\right|^{2 / 3} t\right)-X\left(\left|f^{\prime}(s) / 2\right|^{2 / 3} t\right)$ by $T_{n}(s, t)$. Then, $T_{n}(s, t)$ is the least concave majorant at time zero of

$$
\left\{-\left|\frac{f^{\prime}(s)}{2}\right|^{4 / 3} \eta^{2}+\left|\frac{f^{\prime}(s)}{2}\right|^{1 / 3}\left(W_{s}(\eta+t)-W_{s}(t)\right),|\eta| \leqslant 2 c_{n}\right\} .
$$

Moreover, $\widehat{Z}_{c_{n}}(s)=\widehat{X}_{d_{n}}(0)$ so by Cauchy-Schwarz inequality,

$$
\begin{aligned}
& \left|\operatorname{cov}\left(\left|\widehat{Z}_{c_{n}}(s)\right|^{q},\left|\widehat{Z}_{c_{n}}\left(s+t n^{-1 / 3}\right)\right|^{q}\right)-\operatorname{cov}\left(\left|\widehat{X}_{d_{n}}(0)\right|^{q},\left|T_{n}(s, t)\right|^{q}\right)\right| \\
& \leqslant m_{2 q}^{1 / 2} \mathbb{E}^{1 / 2}\left(\left|\widehat{Z}_{c_{n}}\left(s+t n^{-1 / 3}\right)\right|^{q}-\left|T_{n}(s, t)\right|^{q}\right)^{2} .
\end{aligned}
$$

Assume first $q \leqslant 1$. We have (5.24) with $r=q$ for all positive $x, y$. Moreover, $f^{\prime \prime}$ is assumed to be bounded. By Lemma 2.2, there thus exists some positive $C_{q}$ that does not depend on $s$ or $t$ such that the right hand term in (5.30) is less than or equal to $C_{q}\left(n^{-1 / 3} c_{n}^{2} t\right)^{q}$. Therefore

$$
\begin{gathered}
\lim _{n \rightarrow \infty} n^{\alpha} \sup _{t, s}\left[\operatorname{cov}\left(\left|\widehat{Z}_{c_{n}}\left(s+t n^{-1 / 3}\right)\right|^{q},\left|\widehat{Z}_{c_{n}}(s)\right|^{q}\right)\right. \\
\left.-\operatorname{cov}\left(\left|\widehat{X}_{d_{n}}(0)\right|^{q},\left|T_{n}(s, t)\right|^{q}\right)\right]=0
\end{gathered}
$$

for all $\alpha<q / 3$, where the supremum is taken over $t \in\left[0,4 c_{n}\right], s \in\left[n^{-1 / 4}, 1-n^{-1 / 4}\right]$. From finiteness of $\mathbb{E}|\widehat{X}(0)|^{p}$ for all $p>0$, Lemma 3.1 and the regularity assumptions on $f$, we have

$$
n^{1 / 3} \operatorname{var}\left(\int_{0}^{1} Y_{n}(t) d t\right)=2 \int_{0}^{1} \int_{0}^{4 c_{n}}\left|\frac{f^{\prime}(s)}{2}\right|^{-2 q / 3} \operatorname{cov}\left(\left|\widehat{X}_{d_{n}}(0)\right|^{q},\left|T_{n}(s, t)\right|^{q}\right) d t d s+\mathrm{o}(1) .
$$

Change of variable and Lemma 3.2 finally yields

$$
\lim _{n \rightarrow \infty} n^{1 / 3} \operatorname{var}\left(\int_{0}^{1} Y_{n}(t) d t\right)=\Gamma_{q}^{2}
$$

Assume now $q>1$. We have (5.25) with $r=q$ for all positive $x$ and $y$. There thus exists some positive $C_{q}$ that does not depend on $s$ or $t$ such that the right hand term in (5.30) is less than or equal to $C_{q} n^{-1 / 3} c_{n}^{2} t$, which implies (5.31) for all $\alpha<1 / 3$. Therefore, (5.32) still holds in the case where $q>1$.

For all $n \in \mathbb{N}$, let $L_{n}=n^{-1 / 3}(\log n)^{3}, L_{n}^{\prime}=n^{-1 / 3} \log n$ and let $N_{n}$ denote the integer part of $\left(L_{n}+L_{n}^{\prime}\right)^{-1}$. Let $a_{0}=0$ and for all $n \in \mathbb{N}$ and all $j \in\left\{0, \ldots, N_{n}-1\right\}$, let $a_{2 j+1}=a_{2 j}+L_{n}$ and $a_{2 j+2}=a_{2 j+1}+L_{n}^{\prime}$. Then, the intervals $\left[a_{2 j+1}, a_{2 j+2}\right]$ are the small 
blocs of length $L_{n}^{\prime}$ while the intervals $\left[a_{2 j}, a_{2 j+1}\right]$ are the large blocs of length $L_{n}$. The first derivative of $f$ is bounded away from zero and $\mathbb{E}\left|\widehat{Z}_{c_{n}}(t)\right|^{2 q}$ is uniformly bounded in $n$ and $t$. So there exists some positive $A_{q}$ such that

$$
\mathbb{E}^{1 / 2}\left(n^{1 / 6} \int_{a_{2 N_{n}}}^{1} Y_{n}(t) d t\right)^{2} \leqslant n^{1 / 6}\left|1-a_{2 N_{n}}\right| A_{q} .
$$

By definition, for all $j \in\left\{0, \ldots, N_{n}\right\}, a_{2 j}=j\left(L_{n}+L_{n}^{\prime}\right)$. We thus have $1-L_{n}-L_{n}^{\prime} \leqslant$ $a_{2 N_{n}} \leqslant 1$ and therefore the right hand term in inequality (5.33) converges to zero as $n$ goes to infinity. Let $S_{n}$ be defined by

$$
S_{n}=n^{1 / 6} \sum_{j=0}^{N_{n}-1} \int_{a_{2 j+1}}^{a_{2 j+2}} Y_{n}(t) d t .
$$

The random variables $Y_{n}(t)$ are centered at expectation and therefore

$$
\mathbb{E}\left(S_{n}\right)^{2}=n^{1 / 3} \sum_{i, j} \int_{a_{2 j+1}}^{a_{2 j+2}} \int_{a_{2 i+1}}^{a_{2 i+2}} \operatorname{cov}\left(Y_{n}(t), Y_{n}(s)\right) d t d s .
$$

For all $i \neq j, t \in\left[a_{2 i+1}, a_{2 i+2}\right]$ and $s \in\left[a_{2 j+1}, a_{2 j+2}\right]$, we have $|t-s| \geqslant L_{n}$ so $Y_{n}(t)$ and $Y_{n}(s)$ are independent whenever $n$ is large enough (that is $(\log n)^{8 / 3} \geqslant 4 c_{0}^{1 / 3}$ ). So we have for large enough $n$

$$
n^{1 / 3} \sum_{i \neq j} \int_{a_{2 j+1}}^{a_{2 j+2}} \int_{a_{2 i+1}}^{a_{2 i+2}} \operatorname{cov}\left(Y_{n}(t), Y_{n}(s)\right) d t d s=0 .
$$

Moreover, $\left|\operatorname{cov}\left(Y_{n}(t), Y_{n}(s)\right)\right|$ is uniformly bounded in $n$ and $t$ so there exists some positive $A_{q}^{\prime}$ such that

$$
n^{1 / 3} \sum_{j=0}^{N_{n}-1} \int_{a_{2 j+1}}^{a_{2 j+2}} \int_{a_{2 j+1}}^{a_{2 j+2}} \operatorname{cov}\left(Y_{n}(t), Y_{n}(s)\right) d t d s \leqslant n^{1 / 3} N_{n}\left(L_{n}^{\prime}\right)^{2} A_{q}^{\prime},
$$

which converges to zero as $n$ goes to infinity. Therefore, $\mathbb{E}\left(S_{n}\right)^{2}$ converges to zero as $n$ goes to infinity and it follows from (5.33) that

$$
n^{1 / 6} \int_{0}^{1} Y_{n}(t) d t=n^{1 / 6} \sum_{j=0}^{N_{n}-1} \int_{a_{2 j}}^{a_{2 j+1}} Y_{n}(t) d t+R_{n},
$$

where $\mathbb{E}^{1 / 2}\left(R_{n}\right)^{2}$ converges to zero as $n$ goes to infinity. We shall now use the central limit theorem under Lindeberg condition. The random variables $\xi_{n, j}=n^{1 / 6} \int_{a_{2 j}}^{a_{2 j+1}} Y_{n}(t) d t$ 
are centered at expectation and admit finite variance. Moreover, these random variables are independent whenever $n$ is large enough (that is whenever $(\log n)^{2 / 3} \geqslant 4 c_{0}^{1 / 3}$ ) and by $(5.32)$

$$
\lim _{n \rightarrow \infty} \operatorname{var}\left(\sum_{j=0}^{N_{n}-1} \xi_{n, j}\right)=\Gamma_{q}^{2} .
$$

By Hölder's and Markov's inequalities, for all $\delta>0$

$$
\sum_{j=0}^{N_{n}-1} \mathbb{E}\left(\xi_{n, j}^{2} \mathbb{1}_{\left|\xi_{n, j}\right|>\delta}\right) \leqslant \sum_{j=0}^{N_{n}-1} \mathbb{E}\left(\left|\xi_{n, j}\right|^{3}\right) \delta^{-1} .
$$

Since there exists some $A_{q}^{\prime \prime}>0$ such that $\mathbb{E}\left(\left|\xi_{n, j}\right|^{3}\right) \leqslant A_{q}^{\prime \prime} n^{1 / 2} L_{n}^{3}$, the right hand term of the latter inequality converges to zero as $n$ goes to infinity for all $\delta>0$. By the central limit theorem under Lindeberg condition, we thus have

$$
\sum_{j=0}^{N_{n}-1} \xi_{n, j} \stackrel{\mathcal{D}}{\longrightarrow} \mathcal{N}\left(0, \Gamma_{q}^{2}\right),
$$

which completes the proof of the lemma.

\section{REFERENCES}

[1] H.D. Brunk, Estimation of isotonic regression, in: Nonparametric Techniques in Statistical Inference, Cambridge Univ. Press, 1970, pp. 177-195.

[2] C. Durot, Sharp asymptotics for isotonic regression, Probab. Theory Related Fields 122 (2002) 222-240.

[3] P. Groeneboom, G. Hooghiemstra, H.P. Lopuhaä, Asymptotic normality of the $l_{1}$-error of the grenander estimator, Ann. Statist. 27 (1999) 1316-1347.

[4] J. Huang, J.A. Wellner, Estimation of a monotone density or monotone hazard under random censoring, Scand. J. Statist. 22 (1995) 3-33.

[5] J. Kiefer, J. Wolfowitz, Asymptotically minimax estimation of concave and convexe distribution functions, Z. Wahrsch. Verw. Gebiete 34 (1976) 73-85.

[6] V.N. Kulikov, H.P. Lopuhaä, The limit process of the difference between the empirical distribution function and its concave majorant, Manuscript in preparation, 2002.

[7] B.L.S. Prakasa Rao, Estimation of a unimodal density, Sankhya Ser. A 31 (1969) 23-36.

[8] D. Revuz, M. Yor, Continuous Martingales and Brownian Motion, Springer-Verlag, 1991.

[9] A.I. Sakhanenko, Estimates in the invariance principle, Trudy. Inst. Mat. Sibirsk. Otdel (1972) 27-44.

[10] Y. Wang, The limit distribution of the concave majorant of an empirical distribution function, Statist. Probab. Letters 20 (1994) 81-84. 